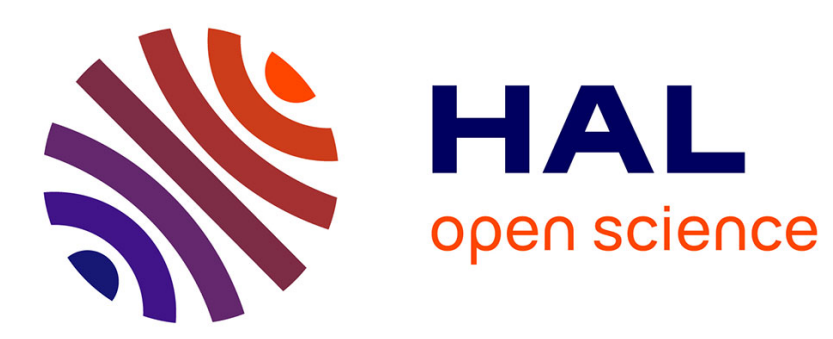

\title{
Some extensions of Pitman's and Ray-Knight's theorems for penalized Brownian motions and their local times, IV
}

Bernard Roynette, Pierre P. Vallois, Marc Yor

\section{To cite this version:}

Bernard Roynette, Pierre P. Vallois, Marc Yor. Some extensions of Pitman's and Ray-Knight's theorems for penalized Brownian motions and their local times, IV. Studia Scientiarum Mathematicarum Hungarica, 2007, 44 (4), pp.469-516. 10.1556/SScMath.2007.1032 . hal-00141528

\section{HAL Id: hal-00141528 \\ https://hal.science/hal-00141528}

Submitted on 13 Apr 2007

HAL is a multi-disciplinary open access archive for the deposit and dissemination of scientific research documents, whether they are published or not. The documents may come from teaching and research institutions in France or abroad, or from public or private research centers.
L'archive ouverte pluridisciplinaire HAL, est destinée au dépôt et à la diffusion de documents scientifiques de niveau recherche, publiés ou non, émanant des établissements d'enseignement et de recherche français ou étrangers, des laboratoires publics ou privés. 


\title{
SOME EXTENSIONS OF PITMAN AND RAY-KNIGHT THEOREMS FOR PENALIZED BROWNIAN MOTIONS AND THEIR LOCAL TIMES, IV
}

\author{
Bernard ROYNETTE ${ }^{(1)},{\text { Pierre } \operatorname{VALLOIS}^{(1)} \text { and Marc YOR }}^{(2),(3)}$
}

November 23, 2005

(1) Université Henri Poincaré, Institut de Mathématiques Elie Cartan, B.P. 239, F-54506 Vandœuvrelès-Nancy Cedex

(2) Laboratoire de Probabilités et Modèles Aléatoires, Universités Paris VI et VII - 4, Place Jussieu - Case 188 - F-75252 Paris Cedex 05.

(3) Institut Universitaire de France.

\begin{abstract}
We show that Pitman's theorem relating Brownian motion and the BES(3) process, as well as the Ray-Knight theorems for Brownian local times remain valid, mutatis mutandis, under the limiting laws of Brownian motion penalized by a function of its one-sided maximum.
\end{abstract}

Key words and phrases : penalization, one-sided maximum, long Brownian bridges, local time, Pitman's theorem, Ray-Knight's theorems.

AMS 2000 subject classifications : 60 G 17, 60 G 40, 60 G 44, 60 J 25, 60 J 35, 60 J 55, 60 J 60 , $60 \mathrm{~J} 65$.

\section{Introduction}

1.1 Let $\left(\Omega=\mathcal{C}\left(\mathbb{R}_{+}, \mathbb{R}\right),\left(X_{t}\right)_{t \geq 0},\left(\mathcal{F}_{t}\right)_{t \geq 0}\right)$ be the canonical space with $\left(X_{t}\right)$ the process of coordinates $: X_{t}(\omega)=\omega(t) ; t \geq 0,\left(\mathcal{F}_{t}\right)_{t \geq 0}$ the canonical filtration associated with $\left(X_{t}\right)$. We write $\mathcal{F}_{\infty}$ for the $\sigma$-algebra generated by $\bigcup_{t \geq 0} \mathcal{F}_{t}$. Let $P_{0}$ be the Wiener measure defined on the canonical space such that $P_{0}\left(X_{0}=0\right)=1$. For any $\lambda \in \mathbb{R}, P_{0}^{\lambda}$ will denote the probability measure (in the sequel, we shall often write the abbreviation p.m.) on $\Omega$ such that $\left(X_{t}\right)$ is a Brownian motion with drift $\lambda$ and started at 0 . Namely, under $P_{0}^{\lambda},\left(X_{t}, t \geq 0\right)$ is distributed as $\left(X_{t}+\lambda t, t \geq 0\right)$ under $P_{0}$.

In our study, to a continuous process $\left(Z_{t}\right)$, we associate its one-sided maximum :

$$
S_{t}^{Z}:=\max _{0 \leq u \leq t} Z_{u}, \quad t \geq 0
$$


and

$$
\begin{aligned}
& R_{t}^{Z}:=2 S_{t}^{Z}-Z_{t}, \quad t \geq 0, \\
& J_{t}^{Z}:=\min _{u \geq t} Z_{u}, \quad t \geq 0 .
\end{aligned}
$$

1.2 We first recall Pitman's theorem ([23],[8]) about the three-dimensional Bessel process : denoting by $\left(\mathcal{R}_{t}\right)$ the natural filtration associated with $\left(R_{t}^{X}\right)$, under $P_{0},\left(R_{t}^{X}\right)$ is a $\left(\mathcal{R}_{t}\right)$ three-dimensional Bessel process started at 0 . Moreover the following projection identity holds :

$$
E_{0}\left[f\left(S_{t}^{X}\right) \mid \mathcal{R}_{t}\right]=\Lambda f\left(R_{t}^{X}\right), \quad \text { for any Borel function } f: \mathbb{R}_{+} \rightarrow \mathbb{R}_{+},
$$

where $\Lambda$ is the kernel :

$$
\Lambda f(r)=\frac{1}{r} \int_{0}^{r} f(x) d x, \quad r \geq 0, f \geq 0 .
$$

In other words, conditionally on $\mathcal{R}_{t}$, and $R_{t}^{X}=r$, the variable $S_{t}^{X}$ is uniformly distributed on $[0, r]$. The identity (1.4) plays an important role in many questions relating Brownian motion and the $B E S(3)$ process; in particular, the intertwining relation : $\mathbb{Q}_{t} \Lambda=\Lambda \mathbb{P}_{t}$, where $\mathbb{Q}_{t}$, resp. $\mathbb{P}_{t}$ denotes the semigroup of the $B E S(3)$ process, resp. of Brownian motion, is easily deduced from (1.4). For a general discussion of the intertwining property as well as many examples, see [6]; in relation with Pitman's theorem, the Pitman-Rogers paper [27] is the most appropriate; see also Ph. Biane [4] for further examples.

Pitman's theorem has been extended ([27]) to processes $\left(Y_{t}\right)$ which are either Brownian motion with drift $\lambda$, or diffusion processes $\left(Y_{t}^{\lambda}\right)$ which solve :

$$
Y_{t}=\beta_{t}+\lambda \int_{0}^{t} \tanh \left(\lambda Y_{s}\right) d s,
$$

where $\left(\beta_{t}\right)$ denotes a one-dimensional Brownian motion starting at 0 .

In fact, $\left(Y_{t}^{\lambda}\right)$ is distributed as $\left(\beta_{t}+\lambda \varepsilon t, t \geq 0\right)$ where $\varepsilon$ denotes a symmetric Bernoulli variable, independent of $\left(\beta_{t}, t \geq 0\right)$.

In these two cases, we know from [27] (see also [14]), that the process $\left(R_{t}^{Y}, t \geq 0\right)$ is, in its own filtration, a diffusion with generator :

$$
\frac{1}{2} \frac{d^{2}}{d x^{2}}+\lambda \operatorname{coth}(\lambda x) \frac{d}{d x}
$$

and there exists a projection identity which extends (1.4) (of course, (1.4) is recovered by letting $\lambda \rightarrow 0):$

$$
E_{0}^{\lambda}\left[f\left(S_{t}^{Y}\right) \mid \sigma\left(R_{u}^{Y}, u \leq t\right)\right]=\Lambda^{\lambda} f\left(R_{t}^{Y}\right), \quad f: \mathbb{R}_{+} \rightarrow \mathbb{R}_{+}, \text {Borel }
$$

with

$$
\Lambda^{\lambda} f(r)=\frac{1}{\int_{0}^{r} e^{2 \lambda x} d x} \int_{0}^{r} e^{2 \lambda x} f(x) d x, \quad r \geq 0, f \geq 0 .
$$

Moreover, it is proved in Rogers [26], that the only "nice" diffusions $\left(Y_{t}\right)$ such that $\left(R_{t}^{Y}\right)$ is, in its own filtration, a diffusion process are either Brownian motion, or more generally Brownian motion with drift or the diffusion $\left(Y_{t}^{\lambda}\right)$ presented in (1.6).

Further extensions of Pitman's theorem and many related discussions have been given in [31], following [33], [34], [18], [24], [32].

One goal of the present paper is to give an extension of the previous results, by defining a larger class of continuous processes $\left(Y_{t}\right)$ such that $R_{t}^{Y}$ is, in its own filtration, a diffusion process. According to Rogers' result, in order to be able to obtain some new extensions of Pitman's theorem, it is now necessary to consider non-Markovian processes $\left(Y_{t}\right)$. Let us reformulate the question in the setting of the canonical space : we are looking for p.m.'s $Q$ on $\left(\Omega, \mathcal{F}_{\infty}\right)$ such that 
1. the two-dimensional process $\left(\left(X_{t}, S_{t}^{X}\right), t \geq 0\right)$ is a $\left(\left(\mathcal{F}_{t}\right), Q\right)$ Markov process; we then say that $\left(X_{t}\right)$ is max-markovian,

2. $\left(R_{t}^{X}\right)$ is a $\left(\left(\mathcal{R}_{t}\right), Q\right)$ diffusion.

We consider p.m.'s $Q$ which are locally absolutely continuous with respect to $P_{0}$, associated with a non-negative $\left(\left(\mathcal{F}_{t}\right), P_{0}\right)$ martingale $\left(M_{t}\right)$, normalized by the condition $M_{0}=1$. Precisely :

$$
Q\left(\Gamma_{t}\right)=E_{0}\left[1_{\Gamma_{t}} M_{t}\right], \quad \forall t \geq 0, \Gamma_{t} \in \mathcal{F}_{t} .
$$

Observe that for any non-negative functional $F$ we have :

$$
E_{Q}\left[F\left(R_{u}^{X}, u \leq t\right)\right]=E_{0}\left[F\left(R_{u}^{X}, u \leq t\right) M_{t}\right]=E_{0}\left[F\left(R_{u}^{X}, u \leq t\right) E_{0}\left[M_{t} \mid \mathcal{R}_{t}\right]\right] .
$$

Obviously, $\left(E_{0}\left[M_{t} \mid \mathcal{R}_{t}\right], t \geq 0\right)$ is a $\left(\left(\mathcal{R}_{t}\right), P_{0}\right)$ martingale.

We assume in the sequel, that $\left(M_{t}\right)$ belongs to one of the four following families of $\left(\left(\mathcal{F}_{t}\right), P_{0}\right)$ martingales .

1. CASE 1. Let $M_{t}=f\left(X_{t}, S_{t}^{X}\right), t \geq 0$, with $f: \mathbb{R} \times \mathbb{R}_{+} \mapsto \mathbb{R}_{+}$a function of class $C^{2,1}$. Then $\left(M_{t}\right)$ coincides with a $P_{0}$-martingale of the type $\left(M_{t}^{\varphi}\right)$ where,

$$
M_{t}^{\varphi}:=\left(S_{t}^{X}-X_{t}\right) \varphi\left(S_{t}^{X}\right)+1-\Phi\left(S_{t}^{X}\right),
$$

$\varphi: \mathbb{R}_{+} \mapsto \mathbb{R}_{+}$satisfies : $\int_{0}^{\infty} \varphi(y) d y=1$, and $\Phi$ is the function : $\Phi(y)=\int_{0}^{y} \varphi(z) d z$.

The class of $P_{0}$-martingales $\left(M_{t}^{\varphi}\right)$ plays a prominent role in Azéma-Yor's solution of Skorokhod's problem studied in [1] (see also [21], [20] [19] for recent developments).

We denote by $Q_{0}^{\varphi}$ the p.m. associated with the martingale density $\left(M_{t}^{\varphi}\right)$ :

$$
Q_{0}^{\varphi}\left(\Gamma_{t}\right)=E_{0}\left[1_{\Gamma_{t}} M_{t}^{\varphi}\right], \quad \forall t \geq 0, \Gamma_{t} \in \mathcal{F}_{t} .
$$

Relation (1.4) implies that :

$$
E_{0}\left[M_{t}^{\varphi} \mid \mathcal{R}_{t}\right]=E_{0}\left[\left(S_{t}^{X}-X_{t}\right) \varphi\left(S_{t}^{X}\right)+1-\Phi\left(S_{t}^{X}\right) \mid \mathcal{R}_{t}\right]=1 .
$$

Therefore $\left(R_{t}^{X}\right)$ is a $\left(\left(\mathcal{R}_{t}\right), Q_{0}^{\varphi}\right)$ three dimensional Bessel process started at 0 (see Theorem 2.1 below).

Our extensions of Pitman's theorem and Ray-Knight's theorems on local times relative to the canonical process under $Q_{0}^{\varphi}$ are stated in Section 2, respectively as Theorem 2.4 on one hand, and Theorems 2.10, 2.11, and 2.13 on the other hand.

2. CASE 2. We choose $M_{t}=e^{-\lambda^{2} t / 2} f\left(X_{t}, S_{t}^{X}\right), t \geq 0$ with $\lambda>0$ and $f: \mathbb{R} \times \mathbb{R}_{+} \mapsto \mathbb{R}_{+}$of class $C^{2,1}$. It is proved in Remark 3.6 in Section 3 that these $P_{0}$-martingales belong to the family $\left\{\left(M_{t}^{\lambda, \varphi}\right)\right\}$ of Kennedy [11] martingales, see also, [25], p 265. These martingales were used in [1] and play a central role in [28]. Let us briefly recall the definition of these processes : to $\psi:[0, \infty[\mapsto[0, \infty[$, a Borel function satisfying :

$$
\int_{0}^{\infty} \psi(z) e^{-\lambda z} d z \leq 1
$$

we associate the function $\Phi: \mathbb{R}_{+} \mapsto \mathbb{R}$ :

$$
\Phi(y)=1-e^{\lambda y}\left(1-\int_{0}^{y} \psi(z) e^{-\lambda z} d z\right), y \geq 0
$$

(we have chosen $\kappa=1-\int_{0}^{\infty} \psi(z) e^{-\lambda z} d z$ in (3.8) of [28]). 
Let $\varphi$ be an a.e. (i.e. w.r.t. the Lebesgue measure) derivative of $\Phi$; then,

$$
\varphi(y):=\Phi^{\prime}(y)=\psi(y)-\lambda e^{\lambda y}\left(1-\int_{0}^{y} \psi(z) e^{-\lambda z} d z\right) .
$$

To $\varphi$ and $\lambda>0$ we associate

(a) the positive $\left(\left(\mathcal{F}_{t}\right), P_{0}\right)$-martingale :

$$
M_{t}^{\lambda, \varphi}:=\left\{\left(1-\Phi\left(S_{t}\right)\right) \cosh \left(\lambda\left(S_{t}-X_{t}\right)\right)+\varphi\left(S_{t}\right) \frac{\sinh \left(\lambda\left(S_{t}-X_{t}\right)\right)}{\lambda}\right\} e^{-\lambda^{2} t / 2},
$$

(b) the p.m. $Q_{0}^{\lambda, \varphi}$ on $\left(\Omega, \mathcal{F}_{\infty}\right)$ characterized by :

$$
Q_{0}^{\lambda, \varphi}\left(\Gamma_{t}\right)=E_{0}\left[1_{\Gamma_{t}} M_{t}^{\lambda, \varphi}\right], \quad \forall t \geq 0, \Gamma_{t} \in \mathcal{F}_{t} .
$$

Therefore, assuming that $\left(M_{t}\right)$ is equal to $\left(M_{t}^{\lambda, \varphi}\right)$ for some $\lambda>0$ and $\varphi$ as above, an easy computation, which is made in [30], subsection 2.3, equation (23), yields to :

$$
E_{0}\left[M_{t} \mid \mathcal{R}_{t}\right]=\frac{\sinh \left(\lambda R_{t}^{X}\right)}{\lambda R_{t}^{X}} e^{-\lambda^{2} t / 2}, \quad t \geq 0
$$

This allows us to prove (see Theorem 3.5 below for a precise formulation) that $\left(R_{t}^{X}\right)$ is a $\left(\left(\mathcal{R}_{t}\right), Q_{0}^{\lambda, \varphi}\right)$ diffusion distributed as the process $\left(Y_{t}^{\lambda}\right)$ defined in (1.6).

Pitman and Ray-Knight's theorems under $Q_{0}^{\lambda, \varphi}$ are presented in Section 3, as Theorems 3.5 and 3.7 respectively.

3. CASE 3. Roughly speaking, Case 3 is the version of Case 1 where the one-sided maximum is replaced by the local time process at $0,\left(L_{t}, t \geq 0\right)$. More precisely let $\left.h: \mathbb{R}_{+} \mapsto\right] 0, \infty[$ be a Borel function satisfying $\int_{0}^{\infty} h(y) d y=1$, and $H(x)=\int_{0}^{x} h(y) d y, x \geq 0$. Thanks to [10] or [25] (Chap. VI) the process : $M_{t}^{h}:=\left|X_{t}\right| h\left(L_{t}^{0}\right)+1-H\left(L_{t}^{0}\right), t \geq 0$ is a $\left(\left(\mathcal{F}_{t}\right), P_{0}\right)$ martingale. Note that the relationship between the $\left(M_{t}^{\varphi}\right)$-martingale defined in (1.12) and the previous $\left(M_{t}^{h}\right)$ martingales comes from Lévy's theorem, since under $P_{0},\left(\left(S_{t}^{X}-X_{t}, S_{t}^{X}\right), t \geq 0\right)$ and $\left(\left(\left|X_{t}\right|, L_{t}^{0}\right), t \geq 0\right)$ have the same distribution.

Let $\widehat{Q}_{0}^{h}$ be the p.m. on the canonical space :

$$
\widehat{Q}_{0}^{h}\left(\Gamma_{t}\right):=E_{0}\left[1_{\Gamma_{t}} M_{t}^{h}\right], \quad \text { for every } t \geq 0 \text { and } \Gamma_{t} \in \mathcal{F}_{t} .
$$

In Section 4, we present and prove an extension of Pitman's theorem, resp. Ray-Knight's theorem in Theorem 4.3, resp. in Theorems 4.4, 4.6 and 4.7.

4. CASE 4. We consider more generally than in the above Case $2, P_{0}$ martingales $\left(M_{t}\right)$ of the form $f\left(X_{t}, S_{t}^{X}\right) \exp \left\{-\frac{1}{2} \int_{0}^{t} l\left(R_{u}^{X}\right) d u\right\}$, where $f: \mathbb{R} \times \mathbb{R}_{+} \mapsto \mathbb{R}_{+}$of class $C^{2,1}, l: \mathbb{R}_{+} \mapsto \mathbb{R}_{+}$is bounded and continuous, $f$ and $l$ being related by :

$$
\frac{\partial^{2}}{\partial x^{2}} f(x, y)=l(2 y-x) f(x, y), \quad \forall y \geq x_{+} \quad \text { and } \quad \frac{\partial}{\partial y} f(y, y)=0, \quad \forall y \geq 0 .
$$

If $l$ is of class $C^{1}$, the previous differential system in $f$ may be solved explicitly, see Proposition 2.13 of [30]. Here, we restrict ourselves to martingales $\left(N_{t}^{F}\right)$ of the type

$$
N_{t}^{F}:=\exp \left\{-F\left(2 S_{t}^{X}-X_{t}\right)+2 F\left(S_{t}^{X}\right)-\frac{1}{2} \int_{0}^{t}\left(F^{\prime 2}-F^{\prime \prime}\right)\left(2 S_{u}^{X}-X_{u}\right) d u\right\},
$$


where $F$ is a function of class $C^{2}$.

Let $Q_{0}^{F}$ be the p.m. on the canonical space with local Radon-Nikodym density $\left(N_{t}^{F}\right)$ with respect to $P_{0}$. In this general setting we prove in Theorem 5.4 that under $Q_{0}^{F},\left(R_{t}^{X}\right)$ is in its own filtration a Brownian motion with a drift function which we compute explicitly. In Section 5 we also investigate a converse to the previous result: see Theorem 5.12 for a more precise formulation.

At this point, it is interesting to evoke a different extension of Pitman's theorem given by Matsumoto and Yor in ([13], Thm 1.6), [14] and [15]; see also [16], [17]. Let

$$
Z_{t}=\exp \left(-X_{t}\right) \int_{0}^{t} e^{2 X_{s}} d s ; \quad t \geq 0 .
$$

The authors proved that $\left(Z_{t}\right)$ is, under $P_{0}^{\mu}$, and with respect to its own filtration, a Markov process with generator :

$$
\frac{1}{2} z^{2} \frac{d^{2}}{d z^{2}}+\left(\left(\frac{1}{2}-\mu\right) z+\frac{K_{1+\mu}}{K_{\mu}}\left(\frac{1}{z}\right)\right) \frac{d}{d z},
$$

where $K_{\alpha}$ denotes the Bessel-Mc Donald function with index $\alpha$.

Moreover the following projection relation holds :

$$
\left.E_{0}^{\mu}\left[f\left(X_{t}\right) \mid \mathcal{Z}_{t}\right)\right]=\mathbb{K}^{\mu} f\left(Z_{t}\right), \quad f: \mathbb{R} \rightarrow \mathbb{R}_{+}, \text {Borel, }
$$

where $\left(\mathcal{Z}_{t}\right)$ denotes the natural filtration generated by $\left(Z_{t}\right)$, and $\mathbb{K}^{\mu}$ is the kernel :

$$
\mathbb{K}^{\mu} f(z)=\frac{1}{2 K_{\mu}(1 / z)} \int_{\mathbb{R}} f(x) e^{\mu x} e^{-\cosh x / z} d x .
$$

This result may be interpreted as an extension of Pitman's theorem since :

- The process $\left(Z_{t}\right)$ is a Markov process in its own filtration,

- the loss of information (i.e. $\mathcal{Z}_{t} \subsetneq \mathcal{F}_{t}$ ) shows up in (1.22),

- Pitman's theorem and its extension to Brownian motions with drifts may be recovered from Matsumoto-Yor's theorem in the following limiting procedure : for any $c>0$, it follows from (1.21) that under $P_{0}^{\mu}$, the process :

$$
c \ln \left(\int_{0}^{t} \exp \left\{\frac{2}{c} X_{s}\right\} d s\right)-X_{t}-c \ln \left(c^{2}\right), t>0
$$

is a Brownian motion with "drift" :

$$
b^{(\mu), c}(x)=\frac{d}{d x}\left(\ln \left\{K_{\mu c}\left(e^{-x / c}\right)\right\}\right) .
$$

Therefore, one recovers, from an elementary Laplace method argument, by letting $c \rightarrow 0$, that, under $P_{0}^{\mu},\left(2 S_{t}^{X}-X_{t}, t \geq 0\right)$ is a Brownian motion with "drift" $b^{(\mu)}$ where :

$$
b^{(\mu)}(x)=\lim _{c \downarrow 0} b^{(\mu), c}(x)=\mu \operatorname{coth}(\mu x) .
$$

We mention that, in the present paper, we do not discuss any deeper the Matsumoto-Yor theorem related to (1.21), but, nonetheless our computations in Section 5 may be seen as some deterministic counterpart of some computations for exponential Brownian functionals made in [13], [14], [15].

The rest of the paper consists in the precise statement and proofs, in Section 2,3,4 and 5, of the various extensions of Pitman's theorem and the Ray-Knight theorems for Brownian local times, as summarized in the above discussion of Cases 1,2,3 and 4 above. A tiny Section 6 concludes.

We also mention that an announcement, largely without proofs, of the main results contained in the present paper has been made in [30], which the reader may consult for a quick overview.

Acknowledgment : The authors are grateful to the referee for indicating a number of improvements throughout the paper. 


\section{Generalizations of Pitman and Ray-Knight theorems with the help of the $M^{\varphi}$ martingales}

\subsection{Some results about the $Q_{0}^{\varphi}$ family of p.m.'s}

We keep the notation given in Section 1.

Let $\varphi: \mathbb{R}_{+} \mapsto \mathbb{R}_{+}$satisfy

$$
\int_{0}^{\infty} \varphi(y) d y=1
$$

and denote $\Phi(y)=\int_{0}^{y} \varphi(x) d x, y \geq 0$.

In [28], we have proved that the p.m.'s $Q_{0}^{\varphi}$ may be obtained by a penalization procedure :

$$
Q_{0}^{\varphi}\left(\Gamma_{u}\right):=\lim _{t \rightarrow \infty} \frac{E_{0}\left[1_{\Gamma_{u}} \varphi\left(S_{t}^{X}\right)\right]}{E_{0}\left[\varphi\left(S_{t}^{X}\right)\right]},
$$

for every $u \geq 0$, and $\Gamma_{u}$ in $\mathcal{F}_{u}$.

We recall now how the law of $\left(X_{t}\right)$, under $Q_{0}^{\varphi}$, has been described in [28].

Theorem 2.1 The probability $Q_{0}^{\varphi}$ may be disintegrated as follows :

1. Under $Q_{0}^{\varphi}, S_{\infty}^{X}$ is finite a.s., and admits $\varphi$ as a probability density;

2. Under $Q_{0}^{\varphi}$, conditionally on $S_{\infty}^{X}=y$, the law of $\left(X_{t}\right)$ is equal to $Q_{0}^{(y)}$, where, for any $y>0$, the p.m. $Q_{0}^{(y)}$ on the canonical space is defined as follows :

(a) $\left(X_{t} ; t \leq T_{y}\right)$ is a Brownian motion started at 0 , and considered up to $T_{y}$, its first hitting time of $y$,

(b) the process $\left(X_{T_{y}+t} ; t \geq 0\right)$ is a "three dimensional Bessel process below $y$ ", namely: $\left(y-X_{T_{y}+t} ; t \geq 0\right)$ is a three dimensional Bessel process started at 0.

(c) the processes $\left(X_{t} ; t \leq T_{y}\right)$ and $\left(X_{T_{y}+t} ; t \geq 0\right)$ are independent.

3. Consequently :

$$
\begin{aligned}
& Q_{0}^{\varphi}\left(\Gamma \mid S_{\infty}^{X}=y\right):=Q_{0}^{(y)}(\Gamma), \quad \text { for any } \Gamma \in \mathcal{F}_{\infty} \\
& Q_{0}^{\varphi}(\cdot)=\int_{0}^{\infty} Q_{0}^{(y)}(\cdot) \varphi(y) d y
\end{aligned}
$$

Remark 2.2 1. In [29] another description of the p.m. $Q_{0}^{(y)}$ has been obtained:

$$
Q_{0}^{(y)}\left(\Gamma_{u}\right)=e^{-y^{2} / 2 u} \sqrt{\frac{2}{\pi u}} E_{0}\left[1_{\Gamma_{u}}\left(y-X_{u}\right) \mid S_{u}^{X}=y\right]+E_{0}\left[1_{\Gamma_{u}} 1_{\left\{S_{u}^{X}<y\right\}}\right]
$$

2. Using stochastic calculus yields to : $M_{t}^{\varphi}=1-\int_{0}^{t} \varphi\left(S_{u}^{X}\right) d X_{u}, t \geq 0$, and :

$$
M_{t}^{\varphi}=\mathcal{E}\left(J^{\varphi}\right)(t):=\exp \left\{\int_{0}^{t} J_{u}^{\varphi} d X_{u}-\frac{1}{2} \int_{0}^{t}\left(J_{u}^{\varphi}\right)^{2} d u\right\}, \quad t \geq 0
$$

where $J_{u}^{\varphi}=-\frac{\varphi\left(S_{u}^{X}\right)}{M_{u}^{\varphi}}$ 
Consequently, $\left(X_{t}, S_{t}^{X}\right)$ solves :

$$
X_{t}=B_{t}-\int_{0}^{t} \frac{\varphi\left(S_{u}^{X}\right)}{\left(S_{u}^{X}-X_{u}\right) \varphi\left(S_{u}^{X}\right)+1-\Phi\left(S_{u}^{X}\right)} d u, \quad t \geq 0,
$$

where $\left(B_{t}\right)$ is a $Q_{0}^{\varphi}$ Brownian motion, $B_{0}=0$.

This implies that $\left(X_{t}, S_{t}^{X}\right)$ is a $\left(\left(\mathcal{F}_{t}\right), Q_{0}^{\varphi}\right)$ Markov process, although, under $Q_{0}^{\varphi},\left(X_{t}\right)$ is not Markov in its own filtration.

The stochastic differential equation (2.6) leads us to look for processes $\left(Z_{t}\right)$ satisfying :

$$
Z_{t}=B_{t}-\int_{0}^{t} \frac{g\left(S_{u}^{Z}\right)}{\left(S_{u}^{Z}-Z_{u}\right) g\left(S_{u}^{Z}\right)+1} d u, \quad t \geq 0,
$$

whose laws are equal to $Q_{0}^{\varphi}$, for some $\varphi$.

Proposition 2.3 1. Let $\varphi$ satisfy (2.1) and suppose for simplicity that $\varphi(y)>0$ for any $y \geq 0$. Define $g$ :

$$
g(y)=\frac{\varphi(y)}{1-\Phi(y)}, \quad y \geq 0 .
$$

Then

(a) $g(y)>0$ for any $y>0$, and

$$
\begin{aligned}
& \int_{0}^{y} g(z) d z<\infty, \quad \forall y \geq 0, \\
& \int_{0}^{\infty} g(z) d z=\infty .
\end{aligned}
$$

(b) There exists a $Q_{0}^{\varphi}$ Brownian motion $\left(B_{t}\right)$, with $B_{0}=0$, such that :

$$
X_{t}=B_{t}-\int_{0}^{t} \frac{g\left(S_{u}^{X}\right)}{\left(S_{u}^{X}-X_{u}\right) g\left(S_{u}^{X}\right)+1} d u, \quad t \geq 0 .
$$

2. Conversely let $\left.g: \mathbb{R}_{+} \mapsto\right] 0, \infty[$ be a Borel function satisfying (2.9) and (2.10). Let $\varphi$ denote the function :

$$
\varphi(y)=g(y) \exp \left\{-\int_{0}^{y} g(z) d z\right\}, \quad y \geq 0 .
$$

(a) Then $\varphi$ takes its values in $] 0, \infty[$ and satisfies (2.1), i.e. : it is a probability density on ] $0, \infty[$.

(b) Consider $\left(Z_{t}\right)$ a process solution of (2.11):

$$
Z_{t}=B_{t}-\int_{0}^{t} \frac{g\left(S_{u}^{Z}\right)}{\left(S_{u}^{Z}-Z_{u}\right) g\left(S_{u}^{Z}\right)+1} d u, \quad t \geq 0,
$$

where $\left(B_{t}\right)$ is a Brownian motion, $B_{0}=0$.

Then the law of $\left(Z_{t}\right)$ is $Q_{0}^{\varphi}$. 
Proof. 1) Relation (2.11) is a direct consequence of Theorem 3.6 of [28]. Relations (2.9) and (2.10) follow from :

$$
\int_{0}^{y} g(z) d z=-\ln (1-\Phi(y)), \quad y \geq 0 .
$$

2) Assume that $\varphi$ is defined by (2.12). Then

$$
\int_{0}^{y} \varphi(z) d z=1-\exp \left\{-\int_{0}^{y} g(z) d z\right\}, \quad y \geq 0 .
$$

It is clear that (2.10) implies (2.1). Since $1-\Phi(y)=\exp \left\{-\int_{0}^{y} g(z) d z\right\}$, Girsanov's theorem implies that the law of $\left(Z_{t}\right)$ is $Q_{0}^{\varphi}$.

\subsection{On Pitman's theorem under $Q_{0}^{\varphi}$}

Our first extension of Pitman's theorem is as follows :

Theorem 2.4 1. Under $Q_{0}^{\varphi}$ the process $\left(R_{t}^{X}\right)$ is, in its own filtration, a three dimensional Bessel process started at 0 , which is independent from $S_{\infty}^{X}$.

2. The following projection relation holds

$$
E_{0}^{\varphi}\left[f\left(S_{t}^{X}\right) \mid \mathcal{R}_{t}\right]=\Lambda^{\varphi} f\left(R_{t}^{X}\right), \quad t \geq 0, \text { for any } f: \mathbb{R}_{+} \mapsto \mathbb{R}_{+}, \text {Borel }
$$

where $\left(\mathcal{R}_{t}\right)$ denotes the natural filtration associated with $\left(R_{t}^{X}\right)$, and $\Lambda^{\varphi}$ is the kernel acting on positive Borel functions :

$$
\Lambda^{\varphi} f(r):=\frac{1}{r} \int_{0}^{r}\{(r-z) \varphi(z)+1-\Phi(z)\} f(z) d z, \quad r>0 .
$$

Remark 2.5 1. It is striking that although the law of $\left(\left(X_{t}, S_{t}^{X}\right), t \geq 0\right)$, under $Q^{\varphi}$, depends on the parameter $\varphi$, the distribution of $\left(R_{t}^{X}, t \geq 0\right)$, under $Q_{0}^{\varphi}$, is independent from $\varphi$. The relation (2.13) expresses how the law of $\left(X_{t}, S_{t}^{X}\right)$, for fixed $t$, depends on $\varphi$.

2. We may recover Theorem 2.1 from Theorem 2.4, because $\left(X_{t}\right)$ may be expressed via $\left(R_{t}^{X}\right)$ and $S_{\infty}^{X}$ (see also Lemma 2.7 below). Recall that under $Q_{0}^{\varphi}, S_{\infty}^{X}$ is a finite r.v., independent from $\left(R_{t}^{X}\right)$. Setting $S_{\infty}^{X}=y$, under $Q_{0}^{\varphi}$ we have :

$$
X_{t}=\left\{\begin{array}{cl}
2 J_{t}^{R^{X}}-R_{t}^{X} & \text { if } t \leq \lambda_{y} \\
2 y-R_{t}^{X} & \text { if } t \geq \lambda_{y}
\end{array}\right.
$$

where

$$
\lambda_{y}:=\sup \left\{t \geq 0 ; R_{t}^{X}=y\right\}=\inf \left\{t \geq 0 ; X_{t} \geq y\right\} .
$$

Then point 2. (a) of Theorem 2.1 follows from the version of Pitman's theorem given in point 2. above. Points 2. (b) and (c) of Theorem 2.1 are consequences of the following property : if $\left(R_{t}\right)$ is a three dimensional Bessel process started at 0 and $\lambda_{y}=\sup \left\{t \geq 0 ; R_{t}=y\right\}$, then $\left(R_{\lambda_{y}+t}-y, t \geq 0\right)$ is independent from $\sigma\left(R_{t \wedge \lambda_{y}}, t \geq 0\right)$ and is distributed as $\left(R_{t}\right)$.

Corollary 2.6 Consider $\left(Z_{t}\right)$ a process solution of (2.11), where the function $g$ satisfies (2.9) and (2.10). Then the process $\left(R_{t}^{Z}\right)$ is, in its own filtration, a three dimensional Bessel process started at 0 , and is independent from $S_{\infty}^{Z}$. 
Proof of Theorem 2.4 1) We have already proved that, under $Q_{0}^{\varphi}$, the process $\left(R_{t}^{X}, t \geq 0\right)$ is, in its own filtration, a three dimensional Bessel process started at 0 .

We now prove that, under $Q_{0}^{\varphi},\left(R_{t}^{X}, t \geq 0\right)$ is independent from $S_{\infty}^{X}$. Let $F$ be a non-negative functional, and $f: \mathbb{R}_{+} \mapsto \mathbb{R}_{+}$be a Borel function. Applying (2.4) we get :

$$
E_{0}^{\varphi}\left[F\left(R_{s}^{X}, s \geq 0\right) f\left(S_{\infty}^{X}\right)\right]=\int_{0}^{\infty} Q_{0}^{(y)}\left[F\left(R_{s}^{X}, s \geq 0\right)\right] f(y) \varphi(y) d y .
$$

Since the law of $\left(R_{t}^{X}, t \geq 0\right)$, under $Q_{0}^{\varphi}$, does not depend on $\varphi$, then $\varphi \mapsto E_{0}^{\varphi}\left[F\left(R_{s}^{X}, s \geq 0\right)\right]$ is constant. Consider a sequence $\left(\varphi_{n}\right)$ converging to the Dirac measure at $y_{0}>0$. Replacing $\varphi$ by $\varphi_{n}$, $f$ by 1 in (2.16) and taking $n \rightarrow \infty$ imply that $\left(R_{t}^{X}, t \geq 0\right)$ is distributed under $Q_{0}^{\left(y_{0}\right)}$, as a three dimensional Bessel process started at 0 . In particular $Q_{0}^{\left(y_{0}\right)}\left[F\left(R_{s}^{X}, s \geq 0\right)\right]$ does not depend on $y_{0}$. Therefore (2.16) implies that, under $Q_{0}^{\varphi}, S_{\infty}^{X}$ is independent from $\left(R_{t}^{X}, t \geq 0\right)$.

2) It remains to establish the projection relation (2.13).

Let $t>0, \Gamma_{t} \in \mathcal{R}_{t}$, and $f: \mathbb{R}_{+} \mapsto \mathbb{R}_{+}$Borel. We have :

$$
E_{0}^{\varphi}\left[1_{\Gamma_{t}} f\left(S_{t}^{X}\right)\right]=E_{0}\left[1_{\Gamma_{t}} f\left(S_{t}^{X}\right) M_{t}^{\varphi}\right]=E_{0}\left[1_{\Gamma_{t}} E_{0}\left\{f\left(S_{t}^{X}\right) M_{t}^{\varphi} \mid \mathcal{R}_{t}\right\}\right] .
$$

Applying (1.4), (1.5), (1.12) and $S_{t}^{X}-X_{t}=R_{t}^{X}-S_{t}^{X}$, we obtain :

$$
E_{0}\left[f\left(S_{t}^{X}\right) M_{t}^{\varphi} \mid \mathcal{R}_{t}\right]=\frac{1}{R_{t}^{X}} \int_{0}^{R_{t}^{X}}\left\{\left(R_{t}^{X}-z\right) \varphi(z)+1-\Phi(z)\right\} f(z) d z=\Lambda^{\varphi} f\left(R_{t}^{X}\right) .
$$

At some stage of this work, J. Pitman asked us the following question : is it possible to characterize the p.m.'s $Q$ such that, under $Q,\left(R_{t}^{X}\right)$ is a three dimensional Bessel process started at 0 ? This led us to determine $\left(X_{t}\right)$ such that the law of $\left(R_{t}^{X}\right)$ is, for instance, that of a three dimensional Bessel process started at 0 . Our approach is based on the following observation : although the path transformation $\left(X_{t}\right) \mapsto\left(R_{t}^{X}\right)$ is not invertible, there exists a one-to-one correspondence between $\left(X_{t}\right)$ and $\left(\left(R_{t}^{X}\right),\left(S_{\infty}^{X}\right)\right)$. This result is stated in Lemma 2.7 below and the answer to Pitman's question is given in Proposition 2.8.

Lemma 2.7 Let $\tau^{X}$ be the random time:

$$
\tau^{X}:=\inf \left\{t \geq 0 ; X_{t}=S_{\infty}^{X}\right\} .
$$

Then :

1. on $\left\{t<\tau^{X}\right\}$, we have $S_{t}^{X}=J_{t}^{R^{X}}$ and therefore

$$
X_{t}=2 S_{t}^{X}-R_{t}^{X}=2 J_{t}^{R^{X}}-R_{t}^{X} ;
$$

2. on $\left\{t \geq \tau^{X}\right\}$, we have $S_{t}^{X}=S_{\infty}^{X}$ and $X_{t}=2 S_{\infty}^{X}-R_{t}^{X}$.

3. Moreover :

$$
\tau^{X}:=\inf \left\{t \geq 0 ; J_{t}^{R} \geq S_{\infty}^{X}\right\}=\sup \left\{t \geq 0 ; R_{t}^{X}<S_{\infty}^{X}\right\} .
$$

Proof. We sketch the proof, the details being left to the reader.

We consider $[a, b]$ an excursion interval of $\left(X_{t}\right)$ below its one-sided maximum, that is $: b$ is finite and

$$
\left.X_{a}=S_{a}^{X} ; X_{b}=S_{b}^{X} ; X_{t}<S_{a}, \text { for any } t \in\right] a, b[.
$$

Suppose that $b<\tau^{X}$, then $S_{t}^{X}=J_{t}^{R^{X}}, \forall t \in[a, b]$.

Define $\lambda^{X}:=\sup \left\{t \geq 0 ; X_{t}=S_{\infty}^{X}\right\}$.

Assuming that $\tau^{X} \leq a$, and $b \leq \lambda^{X}$ forces $S_{t}^{X}=J_{t}^{R^{X}}=S_{\infty}^{X}$.

If the set $\left\{t \geq 0 ; X_{t}=S_{\infty}^{X}\right\}$ is not empty, on the interval $\left[\lambda^{X}, \infty\left[\right.\right.$, we have $S_{t}^{X}=S_{\infty}^{X}$, and $J_{t}^{R^{X}} \geq S_{\infty}^{X}$. 
Proposition 2.8 To a three dimensional Bessel process $\left(R_{t}\right)$ started at 0 , and a r.v. $U$ taking its values in $[0,+\infty]$, we associate:

$$
Z_{t}=\left\{\begin{array}{cc}
2 J_{t}^{R}-R_{t} & \text { if } t \leq \tau \\
2 U-R_{t} & \text { if } t \geq \tau
\end{array}\right.
$$

where $\tau=\sup \left\{t \geq 0 ; R_{t}<U\right\}$, and with the convention $\sup \emptyset=0$.

Then $S_{\infty}^{Z}=U$ and $R_{t}^{Z}=R_{t}, t \geq 0$.

Remark 2.9 1. Pitman's theorem corresponds to the case $U=+\infty$.

2. The canonical space equipped with $Q_{0}^{\varphi}: R_{t}=R_{t}^{X}$ and $U=S_{\infty}^{X}$ gives a particular setting for Proposition 2.8. Note that in this case $U$ is independent from $\left(R_{t}\right)$.

\subsection{A reminder about the Ray-Knight Theorems}

We briefly recall the main results concerning squared Bessel processes and the Ray-Knight Theorems for Brownian local times (see for instance [25]).

We will denote by $\left(L_{t}^{x}, t \geq 0, x \in \mathbb{R}\right)$ the local time process of $\left(X_{t}\right)$.

In order to simplify the writing of the expressions of infinitesimal generators below, we shall use the notation $k_{A}$, meaning $k$ on the set $A$, and 0 on $A^{c}$.

1. For any $\delta \geq 0$, the squared Bessel process, starting at $y \geq 0$, with dimension $\delta$, denoted in the sequel $B E S Q_{y}^{\delta}$, is the diffusion process, valued in $[0, \infty[$, with infinitesimal generator :

$$
2 z \frac{d^{2}}{d z^{2}}+\delta \frac{d}{d z}
$$

2. For $\delta, x, y, t \geq 0$, let $B E S Q_{x \rightarrow y}^{\delta, t}$ denote the $\delta$-dimensional squared Bessel bridge from $x$ to $y$ over the time interval $[0, t]$.

3. Let $\left(Z_{t}\right)$ and $\left(Z_{t}^{\prime}\right)$ be two independent squared Bessel processes with dimension $\delta$, resp. $\delta^{\prime}$ and started resp. at $y$ and $y^{\prime}$. Then $\left(Z_{t}+Z_{t}^{\prime}\right)$ is distributed as the squared $\left(\delta+\delta^{\prime}\right)$-dimensional Bessel process started at $y+y^{\prime}$.

4. (First Ray-Knight theorem) Let $a>0$ and $T_{a}=\inf \left\{t \geq 0 ; X_{t}=a\right\}$. Under $P_{0}$, the process $\left(L_{T_{a}}^{a-x} ; x \geq 0\right)$ is a diffusion process with generator :

$$
2 z \frac{d^{2}}{d z^{2}}+2_{\{0 \leq x \leq a\}} \frac{d}{d z},
$$

where $x$ denotes the "time" variable, and $z$ the "position".

This means :

- conditionally on $L_{T_{a}}^{0},\left(L_{T_{a}}^{a-x} ; 0 \leq x \leq a\right)$ and $\left(L_{T_{a}}^{a-x} ; x \geq a\right)$ are independent;

- $\left(L_{T_{a}}^{a-x} ; 0 \leq x \leq a\right)$ is distributed as $B E S Q_{0}^{2}$.

- conditionally on $L_{T_{a}}^{0}=l,\left(L_{T_{a}}^{-x} ; x \geq 0\right)$ is distributed as $B E S Q_{l}^{0}$.

5. (Second Ray-Knight theorem) Let $\tau_{l}=\inf \left\{t \geq 0 ; L_{t}^{0}>l\right\}$, for any $l>0$. Then, under $P_{0}$, $\left(L_{\tau_{l}}^{x} ; x \geq 0\right)$ and $\left(L_{\tau_{l}}^{-x} ; x \geq 0\right)$ are independent and both are distributed as $B E S Q_{l}^{0}$.

6. (Third Ray-Knight-Williams theorem) Let $\left(R_{t}\right)$ denote the three dimensional Bessel process started at 0 . Then, $\left(L_{\infty}^{x}(R), x \geq 0\right)$ is distributed as $B E S Q_{0}^{2}$. 


\subsection{Ray-Knight theorems under $Q_{0}^{\varphi}$}

Applying directly Theorem 2.1 and with the help of the previous reminder, we obtain the following extension of the first Ray-Knight theorem.

Theorem 2.10 Under $Q_{0}^{\varphi}$, conditionally on $S_{\infty}^{X}=y,\left(L_{\infty}^{y-x}, x \geq 0\right)$ is a diffusion with infinitesimal generator:

$$
2 z \frac{d^{2}}{d z^{2}}+\left(4_{\{0 \leq x \leq y\}}+2_{\{x \geq y\}}\right) \frac{d}{d z} .
$$

Next we state a second extension of the first type Ray-Knight theorem.

Theorem 2.11 Let $a>0$. Under $Q_{0}^{\varphi}$, conditionally on $\left\{T_{a}<\infty\right\}$, the process $\left(L_{T_{a}}^{a-x} ; x \geq 0\right)$ is distributed as the diffusion process with generator (2.22), and is therefore distributed as $\left(L_{T_{a}}^{a-x} ; x \geq 0\right)$, under $P_{0}$.

Remark 2.12 Suppose that $\varphi(y)=0$ for $y \leq b$, for some $b \geq 0$. Let $a \leq b$. Then $Q_{0}^{\varphi}\left(T_{a}<\infty\right)=$ $Q_{0}^{\varphi}\left(S_{\infty}^{X}>b\right)=1$. As a result, we may drop $\left\{T_{a}<\infty\right\}$ in Theorem 2.11.

Proof of Theorem 2.11 Let $n>0$ and $F$ be a non-negative functional. Using the definition of $Q_{0}^{\varphi}$ and the optional stopping theorem we have :

$$
\begin{aligned}
E_{0}^{\varphi}\left[F\left(L_{T_{a}}^{a-x} ; x \geq 0\right) 1_{\left\{T_{a}<n\right\}}\right] & =E_{0}\left[F\left(L_{T_{a}}^{a-x} ; x \geq 0\right) 1_{\left\{T_{a}<n\right\}} M_{n}^{\varphi}\right] \\
& =E_{0}\left[F\left(L_{T_{a}}^{a-x} ; x \geq 0\right) 1_{\left\{T_{a}<n\right\}} M_{T_{a}}^{\varphi}\right] \\
& =(1-\Phi(a)) E_{0}\left[F\left(L_{T_{a}}^{a-x} ; x \geq 0\right) 1_{\left\{T_{a}<n\right\}}\right]
\end{aligned}
$$

since, from (1.12), $M_{T_{a}}^{\varphi}=1-\Phi(a)$.

Taking the limit $n \rightarrow \infty$, we obtain :

$$
E_{0}^{\varphi}\left[F\left(L_{T_{a}}^{a-x} ; x \geq 0\right) 1_{\left\{T_{a}<\infty\right\}}\right]=(1-\Phi(a)) E_{0}\left[F\left(L_{T_{a}}^{a-x} ; x \geq 0\right)\right] .
$$

The result now follows immediately, since, in particular, by taking $F=1$, we find $P_{0}^{\varphi}\left(T_{a}<\infty\right)=$ $P_{0}^{\varphi}\left(S_{\infty}^{X}>a\right)=1-\Phi(a)$.

In fact, conditionally on $\left\{T_{a}<\infty\right\}$ under $Q_{0}^{\varphi},\left(X_{u} ; u \leq T_{a}\right)$ is distributed as $\left(X_{u} ; u \leq T_{a}\right)$ under $P_{0}$.

We now give an extension of the second Ray-Knight theorem.

Theorem 2.13 Let $l>0$. Under $Q_{0}^{\varphi}$, conditionally on $\tau_{l}<\infty$ :

1. $\left(L_{\tau_{l}}^{a} ; a \geq 0\right)$ and $\left(L_{\tau_{l}}^{-a} ; a \geq 0\right)$ are independent;

2. $\left(L_{\tau_{l}}^{-a} ; a \geq 0\right)$ is distributed as a $B E S Q_{l}^{0}$;

3. The law of $\left(L_{\tau_{l}}^{a} ; a \geq 0\right)$ is given as follows :

(a) $S_{\tau_{l}}^{X}$ admits

$$
\frac{(t \varphi(t)+1-\Phi(t)) e^{-l / 2 t}}{c t^{2}} 1_{\{t>0\}}
$$

as density function, with $c=\int_{0}^{\infty} \varphi(t)\left(\frac{1}{t}+\frac{2}{l}\right) e^{-l / 2 t} d t$;

(b) conditionally on $S_{\tau_{l}}^{X}=a,\left(L_{\tau_{l}}^{x} ; 0 \leq x \leq a\right)$ is distributed as the $B E S Q_{l \rightarrow 0}^{4, a}$. 
Proof. 1) We proceed as in Theorem 2.11. Let $F_{-}, F_{+}$be two non-negative functionals and $f$ : $\mathbb{R}_{+} \mapsto \mathbb{R}_{+}$. We have :

$$
E_{0}^{\varphi}\left[F_{-}\left(L_{\tau_{l}}^{-a} ; a \geq 0\right) F_{+}\left(L_{\tau_{l}}^{a} ; a \geq 0\right) f\left(S_{\tau_{l}}^{X}\right) 1_{\left\{\tau_{l}<\infty\right\}}\right]=E_{0}\left[F_{-}\left(L_{\tau_{l}}^{-a} ; a \geq 0\right) F_{+}\left(L_{\tau_{l}}^{a} ; a \geq 0\right) f\left(S_{\tau_{l}}^{X}\right) M_{\tau_{l}}^{\varphi}\right] .
$$

Applying (1.12) yields

$$
M_{\tau_{l}}^{\varphi}=\varphi\left(S_{\tau_{l}}^{X}\right)\left(S_{\tau_{l}}^{X}-X_{\tau_{l}}\right)+1-\Phi\left(S_{\tau_{l}}^{X}\right)=g\left(S_{\tau_{l}}^{X}\right)
$$

where

$$
g(x)=x \varphi(x)+1-\Phi(x), x \geq 0 .
$$

Note that $S_{\tau_{l}}^{X}=\inf \left\{a \geq 0 ; L_{\tau_{l}}^{a}=0\right\}$ and recall that under $P_{0},\left(L_{\tau_{l}}^{a} ; a \geq 0\right)$ and $\left(L_{\tau_{l}}^{-a} ; a \geq 0\right)$ are independent. Therefore :

$E_{0}^{\varphi}\left[F_{-}\left(L_{\tau_{l}}^{a} ; a \geq 0\right) F_{+}\left(L_{\tau_{l}}^{-a} ; a \geq 0\right) f\left(S_{\tau_{l}}^{X}\right) 1_{\left\{\tau_{l}<\infty\right\}}\right]=E_{0}\left[F_{-}\left(L_{\tau_{l}}^{-a} ; a \geq 0\right)\right] E_{0}\left[F_{+}\left(L_{\tau_{l}}^{a} ; a \geq 0\right)(f g)\left(S_{\tau_{l}}^{X}\right)\right]$.

2) We now turn to the computation of :

$$
\Delta_{+}:=E_{0}\left[F_{+}\left(L_{\tau_{l}}^{a} ; a \geq 0\right)(f g)\left(S_{\tau_{l}}^{X}\right)\right]
$$

Let $\left(Y_{t}\right)$ be a 0 -dimensional squared Bessel process started at $l>0$. Define $T_{0}$ to be its first hitting time of 0 . Then the density function of $T_{0}$ is $\frac{l e^{-l / 2 t}}{2 t^{2}} 1_{\{t>0\}}$. Moreover (see for instance [22] section $5.3)$, it is well-known that conditionally on $T_{0}=t,\left(Y_{u}, 0 \leq u \leq t\right)$ is distributed as $B \operatorname{ES}_{l \rightarrow 0}^{4, t}$. As a result, with obvious notations, we get :

$$
\Delta_{+}:=\frac{l}{2} \int_{0}^{\infty} f(t) g(t) \mathbb{E}\left[F_{+}\left(B E S Q_{l \rightarrow 0}^{4, t}(u), u \leq t\right)\right] e^{-l / 2 t} \frac{d t}{t^{2}} .
$$

Taking $F_{-}=F_{+}=1$ implies that, under $Q_{0}^{\varphi}$, conditionally on $\tau_{l}<\infty$, the r.v. $S_{\tau_{l}}^{X}$ admits

$$
\frac{1}{c} \frac{g(t) e^{-l / 2 t}}{t^{2}} 1_{\{t>0\}}
$$

as density function, with

$$
c=\int_{0}^{\infty} g(t) e^{-l / 2 t} \frac{d t}{t^{2}}
$$

Since :

$$
\int_{0}^{\infty}(1-\Phi(t)) e^{-l / 2 t} \frac{d t}{t^{2}}=\int_{0}^{\infty}\left(\int_{t}^{\infty} \varphi(u) d u\right) e^{-l / 2 t} \frac{d t}{t^{2}}=\frac{2}{l} \int_{0}^{\infty} \varphi(u) e^{-l / 2 u} d u
$$

then :

$$
c=\int_{0}^{\infty} \varphi(t)\left(\frac{1}{t}+\frac{2}{l}\right) e^{-l / 2 t} d t
$$

\section{Pitman and Ray-Knight's theorems via the $M^{\lambda, \varphi}$ martin- gales}

\subsection{Some results about the $Q_{0}^{\lambda, \varphi}$ family of p.m.'s}

We keep the notation given in Section $1: \lambda>0, \psi: \mathbb{R} \mapsto] 0,+\infty[$ is a Borel function satisfying (1.14), $\Phi$ is the function associated with $\psi$ via (1.15) and $\varphi=\Phi^{\prime}$. 
In [28] we have proved that the p.m. $Q_{0}^{\lambda, \varphi}$ may be obtained by a penalization principle; let $u>0$ and any $\Gamma_{u}$ in $\mathcal{F}_{u}$, then :

$$
\lim _{t \rightarrow \infty} \frac{E_{0}\left[1_{\Gamma_{u}} \psi\left(S_{t}\right) e^{\lambda\left(S_{t}^{X}-X_{t}\right)}\right]}{E_{0}\left[\psi\left(S_{t}^{X}\right) e^{\lambda\left(S_{t}^{X}-X_{t}\right)}\right]}=E_{0}\left[1_{\Gamma_{u}} M_{u}^{\lambda, \varphi}\right]=Q_{0}^{\lambda, \varphi}\left(\Gamma_{u}\right)
$$

where $\left(M_{u}^{\lambda, \varphi}\right)$ is the $P_{0}$-martingale defined by the relation (1.17).

Let us recall in Theorem 3.1 below the description of the law of $\left(X_{t}\right)$ under $Q_{0}^{\lambda, \varphi}$ which has been determined in ([28], Theorem 4.7).

Theorem 3.1 1. Under $Q_{0}^{\lambda, \varphi}$, the r.v. $S_{\infty}^{X}$ is finite with density function

$$
e^{-\lambda x}\left(\varphi(x)+\lambda(1-\Phi(x))=e^{-\lambda x} \psi(x) .\right.
$$

2. Let $g=\sup \left\{t \geq 0, X_{t}=S_{\infty}^{X}\right\}$. Then $Q_{0}^{\lambda, \varphi}(0<g<\infty)=1$ and under $Q_{0}^{\lambda, \varphi}$ :

(a) $\left(S_{\infty}^{X}-X_{t+g} ; t \geq 0\right)$ is a diffusion independent from $\left(X_{t} ; 0 \leq t \leq g\right)$, with (1.7) as generator.

(b) Conditionally on $S_{\infty}^{X}=x,\left(X_{t} ; t \leq g\right)$ is distributed as a Brownian motion with drift $\lambda$, started at 0 , and stopped when it reaches $x$.

Remark 3.2 1. Theorem 3.1 may be summarized by the following disintegration formula :

$$
Q_{0}^{\lambda, \varphi}(\cdot)=\int_{0}^{\infty} \psi(y) e^{-\lambda y} Q_{0}^{\lambda,(y)}(\cdot) d y,
$$

where $Q_{0}^{\lambda,(y)}$ is the unique p.m. on the canonical space such that:

- $\left(X_{t} ; 0 \leq t \leq T_{y}\right)$ is distributed as a Brownian motion with drift $\lambda$, started at 0 , and stopped at its first hitting time of $y$,

- $\left(y-X_{t+T_{y}} ; t \geq 0\right)$ is distributed as the diffusion with infinitesimal generator (1.7), started at 0 .

Note that (3.2) implies

$$
Q_{0}^{\varphi}=\lim _{\lambda \rightarrow 0} Q_{0}^{\lambda, \varphi} .
$$

2. The p.m. $Q_{0}^{\lambda, \varphi}$ is closely related to the law $P_{0}^{-\lambda}$ of Brownian motion with drift $-\lambda$. More precisely there is the absolute continuity relationship :

$$
Q_{0}^{\lambda, \varphi}=\left(\frac{e^{\lambda S_{\infty}^{X}} \psi\left(S_{\infty}^{X}\right)}{2 \lambda}\right) \cdot P_{0}^{(-\lambda)}
$$

3. $\left(M_{t}^{\lambda, \varphi}\right)$ being a positive $P_{0}$-martingale starting at 1 at time 0 , may be written as an exponential martingale :

$$
M_{t}^{\lambda, \varphi}=\mathcal{E}\left(J^{\lambda, \varphi}\right)_{t},
$$

where :

$$
J_{t}^{\lambda, \varphi}=-\lambda \frac{\varphi\left(S_{t}^{X}\right) \cosh \left(\lambda\left(S_{t}^{X}-X_{t}\right)\right)+\lambda\left(1-\Phi\left(S_{t}^{X}\right)\right) \sinh \left(\lambda\left(S_{t}^{X}-X_{t}\right)\right)}{\lambda\left(1-\Phi\left(S_{t}^{X}\right)\right) \cosh \left(\lambda\left(S_{t}^{X}-X_{t}\right)\right)+\varphi\left(S_{t}^{X}\right) \sinh \left(\lambda\left(S_{t}^{X}-X_{t}\right)\right)} .
$$


As a result, Girsanov's theorem implies that, under $Q_{0}^{\lambda, \varphi}$, the process

$$
\left(X_{t}+\lambda \int_{0}^{t} \frac{\varphi\left(S_{u}^{X}\right) \cosh \left(\lambda\left(S_{u}^{X}-X_{u}\right)\right)+\lambda\left(1-\Phi\left(S_{u}^{X}\right)\right) \sinh \left(\lambda\left(S_{u}^{X}-X_{u}\right)\right)}{\lambda\left(1-\Phi\left(S_{u}^{X}\right)\right) \cosh \left(\lambda\left(S_{u}^{X}-X_{u}\right)\right)+\varphi\left(S_{u}^{X}\right) \sinh \left(\lambda\left(S_{u}^{X}-X_{u}\right)\right)} d u ; t \geq 0\right)
$$

is a Brownian motion, started at 0.

Note that in the calculation of Laplace transforms of sojourn times of Brownian motion in some random interval (see Theorem 2.1 in [12] for details), there appear some expressions similar to the above one.

A result analogous to that of Proposition 2.3 may be obtained in the context of the $Q_{0}^{\lambda, \varphi}$ p.m.'s.

Proposition 3.3 1. Let $\lambda>0$ and $\left.\psi: \mathbb{R}_{+} \mapsto\right] 0, \infty[$ satisfying (1.14). Define $g$ :

$$
g(y)=\frac{\varphi(y)}{1-\Phi(y)}, \quad y \geq 0 .
$$

Then

(a) The function $g$ satisfies :

$$
\begin{aligned}
& g(y)+\lambda>0, \quad y \geq 0, \\
& \int_{0}^{y} g(z) d z<\infty, \quad \forall y \geq 0,
\end{aligned}
$$

(b) There exists a $Q_{0}^{\lambda, \varphi}$ Brownian motion $\left(B_{t}\right)$ such that $B_{0}=0$ and :

$$
X_{t}=B_{t}-\lambda \int_{0}^{t} \frac{g\left(S_{u}^{X}\right) \cosh \left(\lambda\left(S_{u}^{X}-X_{u}\right)\right)+\lambda \sinh \left(\lambda\left(S_{u}^{X}-X_{u}\right)\right)}{\lambda \cosh \left(\lambda\left(S_{u}^{X}-X_{u}\right)+g\left(S_{u}^{X}\right) \sinh \left(\lambda\left(S_{u}^{X}-X_{u}\right)\right)\right.} d u, \quad t \geq 0 .
$$

2. Conversely, let $\left.g: \mathbb{R}_{+} \mapsto\right] 0, \infty[$ be a Borel function satisfying (3.8) and (3.9). Let $\psi$ denote the function:

$$
\psi(y)=(g(y)+\lambda) \exp \left\{-\int_{0}^{y} g(z) d z\right\}, \quad y \geq 0 .
$$

(a) Then $\psi(y)>0$ and (1.14) holds.

(b) Let $\left(Z_{t}\right)$ be a solution of

$$
Z_{t}=B_{t}-\lambda \int_{0}^{t} \frac{g\left(S_{u}^{Z}\right) \cosh \left(\lambda\left(S_{u}^{Z}-Z_{u}\right)\right)+\lambda \sinh \left(\lambda\left(S_{u}^{Z}-Z_{u}\right)\right)}{\lambda \cosh \left(\lambda\left(S_{u}^{Z}-Z_{u}\right)+g\left(S_{u}^{Z}\right) \sinh \left(\lambda\left(S_{u}^{Z}-Z_{u}\right)\right)\right.} d u, \quad t \geq 0,
$$

where $\left(B_{t}\right)$ is a Brownian motion, $B_{0}=0$; then the law of $\left(Z_{t}\right)$ is $Q_{0}^{\lambda, \varphi}$.

Proof. 1) It is clear that (3.10) is a direct consequence of point 3. of Remark 3.2.

The function $\psi$ may be expressed via $g$. Starting with the definition of $g$ and using (1.15), (1.16), we obtain :

$$
g(y)+\lambda=\frac{\psi(y) e^{-\lambda y}}{1-\int_{0}^{y} \psi(z) e^{-\lambda z} d z} \quad y \geq 0 .
$$

Integrating this identity leads to :

$$
1-\int_{0}^{y} \psi(z) e^{-\lambda z} d z=\exp \left\{-\int_{0}^{y}(\lambda+g(z)) d z\right\},
$$


This allows to express $\psi$ as a function of $g$ :

$$
\psi(y)=(g(y)+\lambda) \exp \left\{-\int_{0}^{y} g(z) d z\right\}, \quad y \geq 0 .
$$

Consequently, (3.8) and (3.9) hold.

As for point 2. of Proposition 3.3, assume that $g$ satisfies (3.8) and (3.9). Then

$$
\int_{0}^{y} \psi(z) e^{-\lambda z} d z=1-\exp \left\{-\int_{0}^{y}(\lambda+g(z)) d z\right\}, \quad y \geq 0 .
$$

This proves (1.14).

Applying Girsanov's theorem implies that the law of $\left(Z_{t}\right)$ is $Q_{0}^{\lambda, \varphi}$.

Remark 3.4 1. We now emphasize that the Brownian motion with drift $\lambda$ and the diffusion $\left(Y_{t}\right)$ solving (1.6) may be obtained with the procedure developed in Proposition 3.3.

(a) Let $\psi(y)=0$. Then $\psi$ satisfies (1.14), $\Phi(y)=1-e^{\lambda y}, \varphi(y)=-\lambda e^{\lambda y}$ and $g(y)=-\lambda$. Therefore (3.10) reduces to : $X_{t}=B_{t}+\lambda t, \quad t \geq 0$. This means that:

$$
P_{0}^{(\lambda)}=Q_{0}^{\lambda, \varphi} .
$$

(b) Let $\psi(y)=\lambda e^{-\lambda y}$. Then (1.14) holds, $\Phi(y)=1-\cosh (\lambda y), \varphi(y)=-\lambda \sinh (\lambda y)$, and finally $g(y)=-\lambda \tanh (\lambda y)$. Next, it is easy to check that, under $Q_{0}^{\lambda, \varphi},\left(X_{t}\right)$ solves :

$$
X_{t}=B_{t}+\lambda \int_{0}^{t} \tanh \left(\lambda X_{u}\right) d u, \quad t \geq 0 .
$$

Consequently, the distribution of the diffusion $\left(Y_{t}\right)$, solution of (1.6), is equal to $Q_{0}^{\lambda, \varphi}$ for this particular function $\varphi$.

Note, in this case : $M_{t}^{\lambda, \varphi}=\cosh \left(\lambda X_{t}\right) e^{-\lambda^{2} t / 2}, t \geq 0$.

2. We have already pointed out that Pitman's theorem has been extended to the case where the underlying process $\left(Y_{t}\right)$ is either a Brownian motion with drift $\lambda$ or a diffusion solution of (1.6). Due to the previous point 1., these extensions are actually particular cases of our Theorem 3.1.

3. Under the additional assumption:

$$
\varphi(y)<\lambda(1-\Phi(y)), \quad y \geq 0 .
$$

the drift term in either (3.10) or (3.12) may be simplified.

Since $\varphi(y)+\lambda(1-\Phi(y))=\psi(y)>0$, (3.17) implies that there exists a unique function $\tilde{g}$ such that :

$$
\lambda \tanh (\tilde{g}(y))=g(y)=\frac{\varphi(y)}{1-\Phi(y)}, \quad y \geq 0 .
$$

By an easy calculation, (3.10) may be simplified as follows :

$$
X_{t}=B_{t}-\lambda \int_{0}^{t} \tanh \left(\lambda\left(S_{u}^{X}-X_{u}\right)+\tilde{g}\left(S_{u}^{X}\right)\right) d u, \quad t \geq 0 .
$$

Assume that $\tilde{g}: \mathbb{R}_{+} \mapsto \mathbb{R}$ is given and consider $g(y)=\lambda \tanh \tilde{g}(y)$. Then (3.12) reduces to :

$$
Z_{t}=B_{t}-\lambda \int_{0}^{t} \tanh \left(\lambda\left(S_{u}^{Z}-Z_{u}\right)+\tilde{g}\left(S_{u}^{Z}\right)\right) d u, \quad t \geq 0 .
$$




\subsection{Pitman's theorem under $Q_{0}^{\lambda, \varphi}$}

Our first extension of Pitman's theorem, under $Q_{0}^{\lambda, \varphi}$, is the following.

Theorem 3.5 1. Under $Q_{0}^{\lambda, \varphi}$ the process $\left(R_{t}^{X}\right)$ is, in its own filtration $\left(\mathcal{R}_{t}\right)$, a diffusion started at 0 with generator (1.7), and is independent from $S_{\infty}^{X}$.

2. The following projection relation holds

$$
E_{0}^{\lambda, \varphi}\left[f\left(S_{t}^{X}\right) \mid \mathcal{R}_{t}\right]=\Lambda^{\lambda, \varphi} f\left(R_{t}^{X}\right), \quad t \geq 0,
$$

where

$$
\Lambda^{\lambda, \varphi} f(r):=\frac{\lambda}{\sinh (\lambda r)} \int_{0}^{r}\left\{(1-\Phi(z)) \cosh (\lambda(r-z))+\varphi(z) \frac{\sinh (\lambda(r-z))}{\lambda}\right\} f(z) d z
$$

for any $r>0$ and $f: \mathbb{R}_{+} \mapsto \mathbb{R}_{+}$.

Proof of Theorem 3.5 1) We first prove point 1. of Theorem 3.5.

Let $t>0, F$ be a non-negative functional on $C([0, t])$ and $f: \mathbb{R}_{+} \rightarrow \mathbb{R}_{+}$a Borel function. According to (3.4) and the fact that, under $P_{0}^{(-\lambda)}$, we have $S_{u}^{X}=S_{\infty}^{X}$ for large $u$, then

$$
\begin{aligned}
E_{0}^{\lambda, \varphi}\left[F\left(R_{s}^{X}, s \leq t\right) f\left(S_{\infty}^{X}\right)\right] & =E_{0}^{(-\lambda)}\left[F\left(R_{s}^{X}, s \leq t\right) f\left(S_{\infty}^{X}\right) \frac{e^{\lambda S_{\infty}^{X}}}{2 \lambda} \psi\left(S_{\infty}^{X}\right)\right] \\
& =\lim _{u \rightarrow \infty} E_{0}^{(-\lambda)}\left[F\left(R_{s}^{X}, s \leq t\right) f\left(S_{u}^{X}\right) \frac{e^{\lambda S_{u}^{X}}}{2 \lambda} \psi\left(S_{u}^{X}\right)\right] \\
& =\lim _{u \rightarrow \infty} E_{0}^{(-\lambda)}\left[F\left(R_{s}^{X}, s \leq t\right) E_{0}^{(-\lambda)}\left[f\left(S_{u}^{X}\right) \frac{e^{\lambda S_{u}^{X}}}{2 \lambda} \psi\left(S_{u}^{X}\right) \mid \mathcal{R}_{u}\right]\right]
\end{aligned}
$$

The above conditional expectation can be computed via the projection relations (1.8) and (1.9) :

$$
E_{0}^{(-\lambda)}\left[f\left(S_{u}^{X}\right) \frac{e^{\lambda S_{u}^{X}}}{2 \lambda} \psi\left(S_{u}^{X}\right) \mid \mathcal{R}_{u}\right]=\frac{1}{1-e^{-2 \lambda R_{u}^{X}}} \int_{0}^{R_{u}^{X}} f(x) \psi(x) e^{-\lambda x} d x
$$

Since $P_{0}^{(-\lambda)}\left(\lim _{u \rightarrow \infty} R_{u}^{X}=\infty\right)=1$, we finally get :

$$
E_{0}^{\lambda, \varphi}\left[F\left(R_{s}^{X}, s \leq t\right) f\left(S_{\infty}^{X}\right)\right]=\left(\int_{0}^{\infty} f(x) \psi(x) e^{-\lambda x} d x\right) E_{0}^{(-\lambda)}\left[F\left(R_{s}^{X}, s \leq t\right)\right]
$$

Due to Pitman's theorem under $P_{0}^{(-\lambda)}([27],[14])$, point 1. of Theorem 3.5 follows.

2 ) To prove point 2 . of Theorem 3.5 it is more convenient to use (1.18):

$$
\begin{aligned}
E_{0}^{\lambda, \varphi}\left[F\left(R_{s}^{X}, s \leq t\right) f\left(S_{t}^{X}\right)\right] & =E_{0}\left[F\left(R_{s}^{X}, s \leq t\right) f\left(S_{t}^{X}\right) M_{t}^{\lambda, \varphi}\right] \\
& =E_{0}\left[F\left(R_{s}^{X}, s \leq t\right) E_{0}\left[f\left(S_{t}^{X}\right) M_{t}^{\lambda, \varphi} \mid \mathcal{R}_{t}\right]\right]
\end{aligned}
$$

Combining $X_{t}=2 S_{t}^{X}-R_{t}^{X},(1.4),(1.5)$ and (1.17) we can determine the previous conditional expectation :

$$
E_{0}\left[f\left(S_{t}^{X}\right) M_{t}^{\lambda, \varphi} \mid \mathcal{R}_{t}\right]=f^{\sharp}\left(R_{t}^{X}\right) e^{-\lambda^{2} t / 2},
$$

where

$$
\begin{aligned}
f^{\sharp}(r) & =\frac{1}{r} \int_{0}^{r}\left\{(1-\Phi(z)) \cosh (\lambda(r-z))+\varphi(z) \frac{\sinh (\lambda(r-z))}{\lambda}\right\} f(z) d z \\
& =\frac{\sinh (\lambda r)}{\lambda r} \Lambda^{\lambda, \varphi}(f)(r)
\end{aligned}
$$


(recall that $\Lambda^{\lambda, \varphi} f(r)$ is defined in (3.22)).

Consequently :

$$
E_{0}^{\lambda, \varphi}\left[f\left(S_{t}^{X}\right) \mid \mathcal{R}_{t}\right]=\frac{\Lambda^{\lambda, \varphi} f\left(R_{t}^{X}\right)}{\Lambda^{\lambda, \varphi} 1\left(R_{t}^{X}\right)} .
$$

Integrating by parts and using (1.16), we obtain :

$$
\int_{0}^{r}(1-\Phi(z)) \cosh (\lambda(r-z)) d z=\frac{\sinh (\lambda r)}{\lambda}-\int_{0}^{r} \varphi(z) \frac{\sinh (\lambda(r-z))}{\lambda} d z .
$$

This implies that $\Lambda^{\lambda, \varphi}(1)(r)=1$, ending the proof of Theorem 3.5.

Remark 3.6 1. Using (2.15)implies that Theorem 3.1 may be obtained as a consequence of Theorem 3.5 (see point 3. of Remark 2.5 where an analogous result has been obtained under $Q_{0}^{\varphi}$ ).

2. To $\lambda>0$ and $f: \mathbb{R} \times \mathbb{R}_{+} \mapsto \mathbb{R}$ of class $C^{2,1}$ we associate $: M_{t}:=f\left(X_{t}, S_{t}^{X}\right) e^{-\lambda^{2} t / 2}, t \geq 0$. It is worth pointing out that assuming $\left(M_{t}\right)$ is a $\left(P_{0},\left(\mathcal{F}_{t}\right)_{t \geq 0}\right)$ local martingale implies that $\left(M_{t}\right)$ is a Kennedy martingale (see (1.17) above).

Suppose that $\left(M_{t}\right)$ is a $P_{0}$-local martingale, and $M_{0}=f(0,0)=1$. Then Itô's formula implies that $f$ solves :

$$
\begin{aligned}
& \frac{1}{2} \frac{\partial^{2} f}{\partial x^{2}}(x, y)=\frac{\lambda^{2}}{2} f(x, y), \quad x_{+} \leq y, \\
& \frac{\partial f}{\partial y}(y, y)=0, \quad y \geq 0 .
\end{aligned}
$$

Obviously (3.24) leads to :

$$
f(x, y)=\alpha(y) \cosh (\lambda(y-x))+\varphi(y) \frac{\sinh (\lambda(y-x))}{\lambda} .
$$

Next, $f(0,0)=0$ and (3.25) imply that $\alpha^{\prime}(y)=-\varphi(y)$ and $\alpha(0)=1$. Then $\alpha(y)=1-\Phi(y)=$ $1-\int_{0}^{y} \varphi(z) d z$.

As a result $\left(M_{t}\right)$ is a Kennedy martingale $\left(M^{\lambda, \varphi}\right)$ of the type (1.17).

Note that since $f$ is supposed to be of class $C^{2,1}$, then $\varphi$ is continuous. Note also that $\left(M_{t}^{\lambda, \varphi}\right)$ is a $P_{0}$ local martingale as soon as $\varphi$ is locally bounded. Moreover it is proved in Proposition 3.3 of [28] that $\left(M_{t}^{\lambda, \varphi}\right)$ is a non-negative martingale iff $\varphi$ may be written as (1.16) where the function $\psi$ satisfies the inequality (1.14).

\subsection{Ray-Knight theorems under $Q_{0}^{\lambda, \varphi}$}

Our extension of the first Ray-Knight theorem (see section 2.3 for the terminology) is the following.

Theorem 3.7 Let a be a fixed positive level. Then, under $Q_{0}^{\lambda, \varphi}$, conditionally on $T_{a}<\infty$, the process $\left(L_{T_{a}}^{a-x}, x \geq 0\right)$ is a diffusion process with infinitesimal generator :

$$
2 z \frac{d^{2}}{d z^{2}}+\left(2_{\{0 \leq x \leq a\}}+2 \lambda z\right) \frac{d}{d z} .
$$

Proof. Let $F$ be a non-negative functional on $C([0, \infty[)$. Using (3.4), we get :

$$
\begin{aligned}
E_{0}^{\lambda, \varphi}\left[F\left(L_{T_{a}}^{a-x} ; x \geq 0\right) 1_{\left\{T_{a}<\infty\right\}}\right] & =\frac{1}{2 \lambda} E_{0}^{(-\lambda)}\left[F\left(L_{T_{a}}^{a-x} ; x \geq 0\right) 1_{\left\{T_{a}<\infty\right\}} \psi\left(S_{\infty}^{X}\right) e^{\lambda S_{\infty}^{X}}\right] \\
& =\frac{1}{2 \lambda} E_{0}^{(-\lambda)}\left[F\left(L_{T_{a}}^{a-x} ; x \geq 0\right) 1_{\left\{T_{a}<\infty\right\}} E_{0}^{(-\lambda)}\left\{\psi\left(S_{\infty}^{X}\right) e^{\lambda S_{\infty}^{X}} \mid \mathcal{F}_{T_{a}}\right\}\right] .
\end{aligned}
$$


Since, under $P_{0}^{(-\lambda)}$, conditionally on $T_{a}<\infty,\left(X_{T_{a}+t}, t \geq 0\right)$ is a Brownian motion with drift $-\lambda$, started at level $a$, then $P_{a}^{(-\lambda)}\left(S_{\infty}>a+y\right)=e^{-2 \lambda y}, y \geq 0$.

Consequently, on $\left\{T_{a}<\infty\right\}$, we have

$$
E_{0}^{(-\lambda)}\left\{\psi\left(S_{\infty}^{X}\right) e^{\lambda S_{\infty}^{X}} \mid \mathcal{F}_{T_{a}}\right\}=2 \lambda e^{2 \lambda a} \int_{a}^{\infty} \psi(z) e^{-\lambda z} d z
$$

This implies that under $Q_{0}^{\lambda, \varphi}$, conditionally on $T_{a}<\infty$, the process $\left(L_{T_{a}}^{a-x}, x \geq 0\right)$ is distributed as $\left(L_{T_{a}}^{a-x}, x \geq 0\right)$ under $P_{0}^{(-\lambda)}$.

From Proposition 5 in [9], we conclude that (3.26) holds.

\section{Pitman and Ray-Knight theorems under $\widehat{Q}_{0}^{h}$}

\subsection{Some results about the $\widehat{Q}_{0}^{h}$ family of p.m.'s}

From Lévy's theorem, under $P_{0},\left(\left(S_{t}^{X}-X_{t}, S_{t}^{X}\right), t \geq 0\right)$ and $\left(\left(\left|X_{t}\right|, L_{t}^{0}\right), t \geq 0\right)$ have the same distribution. This implies that :

$$
M_{t}^{h}:=\left|X_{t}\right| h\left(L_{t}^{0}\right)+1-H\left(L_{t}^{0}\right), \quad t \geq 0,
$$

is a $\left(\left(\mathcal{F}_{t}\right), P_{0}\right)$ martingale, where $\left.h: \mathbb{R}_{+} \mapsto\right] 0, \infty\left[\right.$ is Borel and $H(x)=\int_{0}^{x} h(y) d y, x \geq 0$.

In the sequel it is always supposed that $h$ is a density function, i.e.

$$
\int_{0}^{\infty} h(y) d y=1 .
$$

Associated with $h$, let $\widehat{Q}_{0}^{h}$ be the p.m. on the canonical space induced by the relations :

$$
\widehat{Q}_{0}^{h}\left(\Gamma_{t}\right):=E_{0}\left[1_{\Gamma_{t}} M_{t}^{h}\right]
$$

for every $t \geq 0$ and $\Gamma_{t} \in \mathcal{F}_{t}$.

Although it will not be used here, it is worth pointing out that the p.m.'s $\widehat{Q}_{0}^{h}$ may be obtained by a penalization procedure :

$$
\widehat{Q}_{0}^{h}\left(\Gamma_{u}\right)=\lim _{t \rightarrow \infty} \frac{E_{0}\left[1_{\Gamma_{u}} h\left(L_{t}^{0}\right)\right]}{E_{0}\left[h\left(L_{t}^{0}\right)\right]}, \quad \forall u \geq 0, \Gamma_{u} \in \mathcal{F}_{u} .
$$

The next Theorem summarizes the main properties of $\left(X_{t}\right)$ under $\widehat{Q}_{0}^{h}$, obtained in [28].

Theorem 4.1 The probability $\widehat{Q}_{0}^{h}$ may be disintegrated as follows :

1. Under $\widehat{Q}_{0}^{h}, L_{\infty}^{0}$ is finite a.s., and admits $h$ as its probability density;

2. Under $\widehat{Q}_{0}^{h}$, conditionally on $L_{\infty}^{0}=l$, the distribution of $\left(X_{t}\right)$ is equal to $\widehat{Q}_{0}^{(l)}$, where, for any $l>0$, the p.m. $\widehat{Q}_{0}^{(l)}$ on the canonical space is defined as follows :

(a) $\left(X_{u}, u \leq \tau_{l}\right)$ is distributed as a Brownian motion started at 0 and stopped when its local time at 0 first exceeds $l$,

(b) with probability $1 / 2,\left(X_{\tau_{l}+u}, u \geq 0\right)$ (resp. $\left(-X_{\tau_{l}+u}, u \geq 0\right)$ ) is distributed as a three dimensional Bessel process started at 0 . 
3. Consequently :

$$
\begin{aligned}
& \widehat{Q}_{0}^{h}\left(\Gamma \mid L_{\infty}^{0}=l\right):=\widehat{Q}_{0}^{(l)}(\Gamma), \quad \forall \Gamma \in \mathcal{F}_{\infty}, \\
& \widehat{Q}_{0}^{h}(\cdot)=\int_{0}^{\infty} \widehat{Q}_{0}^{(l)}(\cdot) h(l) d l .
\end{aligned}
$$

Remark 4.2 1. In [29], limiting laws for long Brownian bridges perturbed by their one-sided maximum have been investigated. Using Lévy's isomorphism, recalled at the beginning of this subsection, it is possible to obtain an analogous result relative to local time:

$$
\begin{aligned}
& \lim _{t \rightarrow \infty} P_{0}\left(\Gamma_{u} \mid L_{t}^{0}=l\right)=\widehat{Q}_{0}^{(l)}\left(\Gamma_{u}\right), \\
& \widehat{Q}_{0}^{(l)}\left(\Gamma_{u}\right)=e^{-l^{2} / 2 u} \sqrt{\frac{2}{\pi u}} E_{0}\left[1_{\Gamma_{u}}\left|X_{u}\right| \mid L_{u}^{0}=l\right]+E_{0}\left[1_{\Gamma_{u}} 1_{\left\{L_{u}^{0}<l\right\}}\right],
\end{aligned}
$$

for any $u \geq 0, \Gamma_{u} \in \mathcal{F}_{u}$.

2. A more general result than Theorem 4.1 has been given in [28] .

3. Although $\left(X_{t}\right)$ is not Markov under $\widehat{Q}_{0}^{h}$, the pair $\left(X_{t}, L_{t}^{0}\right)$ is Markov and satisfies (cf Theorem 3.13 in [28]) :

$$
X_{t}=B_{t}+\int_{0}^{t} \frac{\operatorname{sgn}\left(X_{s}\right) h\left(L_{s}^{0}\right)}{M_{s}^{h}} d s
$$

where $\left(B_{t}\right)$ is a $\widehat{Q}_{0}^{h}$-Brownian motion started at 0 .

\subsection{Pitman's theorem under $\widehat{Q}_{0}^{h}$}

From Lévy's theorem, it is well-known that Pitman's theorem admits a formulation in terms of local time : under $P_{0},\left(\left|X_{t}\right|+L_{t}^{0}, t \geq 0\right)$ is distributed as the three dimensional Bessel process started at 0 . Let $\left.h: \mathbb{R}_{+} \mapsto\right] 0, \infty[$ satisfying (4.2). According to Theorem 4.1 and using analogous arguments developed in Remark 2.5, our extension of Pitman's theorem is the following.

Theorem 4.3 Under $\widehat{Q}_{0}^{h},\left(\left|X_{t}\right|+L_{t}^{0}, t \geq 0\right)$ is distributed as the three dimensional Bessel process started at 0 and is independent from $L_{\infty}^{0}$.

\subsection{Ray-Knight theorems under $\widehat{Q}_{0}^{h}$}

In this subsection we state an extension of the three Ray-Knight theorems (see Section 2.3 again).

Theorem 4.4 Under $\widehat{Q}_{0}^{h}$, conditionally on $L_{\infty}^{0}=l$,

1. $\left(L_{\infty}^{x}, x \geq 0\right)$ and $\left(L_{\infty}^{-x}, x \geq 0\right)$ are independent,

2. conditionally on: " $X_{t}$ drifts to $+\infty$ as $t \rightarrow \infty$ ",

(a) $\left(L_{\infty}^{x}, x \geq 0\right)$ is distributed as $B E S Q_{l}^{2}$.

(b) $\left(L_{\infty}^{-x}, x \geq 0\right)$ is distributed as $B E S Q_{l}^{0}$.

Proof. From Theorem 4.1 and the second Ray-Knight theorem, conditionally on $L_{\infty}^{0}=l,\left(L_{\tau_{l}}^{x}, x \geq 0\right)$ and $\left(L_{\tau_{l}}^{-x}, x \geq 0\right)$ are independent, and distributed as $B E S Q_{l}^{0}$.

Let $\xi$ denote the process : $\xi_{t}=X_{\tau_{l}+t}, t \geq 0$.

Recall that either $\xi_{t}>0, \forall t>0$ or $\xi_{t}<0, \forall t>0$, and the probability of each of the two previous events is $1 / 2$.

Assume that $\left(\xi_{t}\right)$ remains positive. According to the third Ray-Knight theorem, $\left(L_{\infty}^{x}(\xi), x \geq 0\right)$ is distributed as $B E S Q^{2}$ started at 0 .

The additivity property of squared Bessel processes recalled in subsection 2.3 implies Theorem 4.4. 
Remark 4.5 Thanks to Theorem 4.1, it is clear that $\left(L_{\infty}^{x}, x \geq 0\right)$ and $\left(L_{\infty}^{-x}, x \geq 0\right)$ play symmetric roles. In particular, conditionally on: " $X_{t}$ drifts to $-\infty$ as $t \rightarrow \infty$ ", the process $\left(L_{\infty}^{-x}, x \geq 0\right)$, resp. $\left(L_{\infty}^{x}, x \geq 0\right)$, is distributed as $B E S Q_{l}^{2}$, resp. BESQ $Q_{l}^{0}$.

We now state an extension of the second Ray-Knight theorem whose proof makes no appeal to Theorem 4.1 .

Theorem 4.6 Conditionally on $L_{\infty}^{0}>l$, under $\widehat{Q}_{0}^{h},\left(L_{\tau_{l}}^{x}, x \geq 0\right)$ and $\left(L_{\tau_{l}}^{-x}, x \geq 0\right)$ are independent and both are distributed as the $B E S Q_{0}^{2}$ process.

Proof. Mimicking the proof of Theorem 2.11, we have

$$
\widehat{E}_{0}^{h}\left[F_{+}\left(L_{\tau_{l}}^{x}, x \geq 0\right) F_{-}\left(L_{\tau_{l}}^{-x}, x \geq 0\right) 1_{\left\{\tau_{l}<\infty\right\}}\right]=E_{0}\left[F_{+}\left(L_{\tau_{l}}^{x}, x \geq 0\right) F_{-}\left(L_{\tau_{l}}^{-x}, x \geq 0\right) M_{\tau_{l}}^{h}\right],
$$

where $F_{ \pm}$are non-negative functionals on $C([0, \infty[)$.

Since $M_{\tau_{l}}^{h}=1-H(l)=\widehat{Q}_{0}^{h}\left(L_{\infty}^{0}>l\right)$, the second Ray-Knight theorem for Brownian motion implies Theorem 4.6.

Theorem 4.7 Let $a>0$. Under $\widehat{Q}_{0}^{h}$, conditionally on $T_{a}<\infty,\left(L_{T_{a}}^{a-x}, x \geq 0\right)$ is an inhomogeneous Markov process with infinitesimal generator :

$$
\mathcal{L}^{h, x} f(z)=2 z f^{\prime \prime}(z)+\left(4 z \frac{\partial}{\partial z} \ln \tilde{\theta}(x, z)+2\right) 1_{\{0 \leq x \leq a\}} f^{\prime}(z),
$$

where

$$
\tilde{\theta}(x, z)=\frac{1}{2(a-x)} e^{-z / 2(a-x)} \int_{0}^{\infty}(a h(y)+1-H(y)) e^{-y / 2(a-x)} I_{0}\left(\frac{\sqrt{z y}}{2(a-x)}\right) d y, \quad 0 \leq x<a, z \geq 0 .
$$

Proof. Let $F_{ \pm}$be non-negative functionals on $C\left(\left[0, \infty[)\right.\right.$. From the definition of $\widehat{Q}_{0}^{h}$ we easily obtain

$$
\begin{aligned}
\widehat{E}_{0}^{h}\left[F_{+}\left(L_{T_{a}}^{a-x}, 0 \leq x \leq a\right) F_{-}\left(L_{T_{a}}^{-x}, x \geq 0\right) 1_{\left\{T_{a}<\infty\right\}}\right] & =E_{0}\left[F_{+}\left(L_{T_{a}}^{a-x}, 0 \leq x \leq a\right) F_{-}\left(L_{T_{a}}^{-x}, x \geq 0\right) M_{T_{a}}^{h}\right] \\
& =E_{0}\left[F_{+}\left(L_{T_{a}}^{a-x}, 0 \leq x \leq a\right) F_{-}\left(L_{T_{a}}^{-x}, x \geq 0\right) \theta\left(L_{T_{a}}^{0}\right)\right]
\end{aligned}
$$

where $\theta(l)=a h(l)+1-H(l), l \geq 0$.

By the first Ray-Knight theorem, conditionally on $L_{T_{a}}^{0}=l,\left(L_{T_{a}}^{a-x}, 0 \leq x \leq a\right)$ and $\left(L_{T_{a}}^{-x}, x \geq 0\right)$ are independent and the second one is distributed as a $B E S Q_{l}^{0}$. Thus it remains to study the law of $\left(Y(z):=L_{T_{a}}^{a-x}, 0 \leq x \leq a\right)$.

Recall that, under $P_{0}$, the law of $(Y(x), x \leq a)$ is $\mathbb{Q}_{0}^{2}$, where $\mathbb{Q}_{l}^{2}$ denotes the law of the $B E S Q_{l}^{2}$ process. It is clear that :

$$
\widehat{E}_{0}^{h}\left[F_{+}(Y(x), 0 \leq x \leq a) 1_{\left\{T_{a}<\infty\right\}}\right]=\mathbb{Q}_{0}^{2}\left[F_{+}(Y(x), 0 \leq x \leq a) \theta(Y(a))\right] .
$$

This identity implies that:

$$
\widehat{E}_{0}^{h}\left[f(Y(c)) \mid \sigma(Y(x), 0 \leq x \leq b) \vee \sigma\left(\left\{T_{a}<\infty\right\}\right)\right]=\widetilde{\mathbb{Q}}^{2}(b, c ; f)(Y(c)),
$$

where for $f: \mathbb{R}_{+} \mapsto \mathbb{R}_{+}$, and $0 \leq b<c<a$,

$$
\widetilde{\mathbb{Q}}^{(2)}(b, c ; f)(y)=\frac{\mathbb{Q}_{y}^{2}[f(Y(c-b)) \tilde{\theta}(c, Y(c-b))]}{\tilde{\theta}(c, y)},
$$


and

$$
\tilde{\theta}(c, y)=\mathbb{Q}_{y}^{2}[\theta(Y(a-c))], \quad 0 \leq c \leq a .
$$

Let $q^{(2)}(c ; y, z)$ denote the transition probabilities of $B E S Q^{2}$, then :

$$
\begin{aligned}
\tilde{\theta}(c, y) & =\int_{0}^{\infty} \theta(z) q^{(2)}(a-c ; y, z) d z \\
& =\frac{1}{2(a-c)} e^{-y / 2(a-c)} \int_{0}^{\infty} \theta(z) e^{-z / 2(a-c)} I_{0}\left(\frac{\sqrt{z y}}{2(a-c)}\right) d z
\end{aligned}
$$

The function $(c, y) \mapsto \tilde{\theta}(c, y)$ is a space-time harmonic function (defined on $[0, a] \times \mathbb{R}_{+}$) with respect to $\left(Y(x)\right.$ ). Since the generator of $(Y(x))$ is given by (2.21) (with $d=2$ ), therefore, under $\widehat{Q}_{0}^{h}$, the process $(Y(z), 0 \leq z \leq a)$ is an inhomogeneous Markov process, with infinitesimal generator :

$$
2 y \frac{d^{2}}{d y^{2}}+\left(4 y \frac{\partial}{\partial y} \ln \tilde{\theta}(x, y)+2\right) \frac{d}{d y},
$$

where $x$ denotes "time" and $y$ the space variable.

\section{A class of max-diffusions which enjoy Pitman's property}

Let us introduce the following functional spaces :

$$
\begin{aligned}
& \mathcal{H}=\{F:] 0, \infty\left[\mapsto \mathbb{R}, F \text { of class } C^{1}, \int_{0}^{x} e^{2 F(y)} d y<\infty, \forall x \in \mathbb{R}_{+}\right\}, \\
& \mathcal{H}^{\infty}=\left\{F \in \mathcal{H} ; \int_{0}^{\infty} e^{2 F(y)} d y=\infty\right\} .
\end{aligned}
$$

We define $T$ the non-linear operator from $\mathcal{H}$ to be :

$$
T F(x)=-F^{\prime}(x)+\frac{e^{2 F(x)}}{\int_{0}^{x} e^{2 F(y)} d y}, \quad x>0, F \in \mathcal{H} .
$$

Note that $T(F+c)=T(F)$, where $c$ is a constant.

The aim of this section is to give a large class of max-diffusion processes $\left(Y_{t}\right)$ such that $\left(R_{t}^{Y}=\right.$ $2 S_{t}^{Y}-Y_{t}, t \geq 0$ ) is a diffusion in its own filtration. We will prove (see Theorem 5.4 below) that $\left(R_{t}^{Y}, t \geq 0\right)$ is a diffusion with drift coefficient $T F$, for some $F \in \mathcal{H}$ associated with $\left(Y_{t}\right)$. This leads us to study, in a preliminary step, diffusions $\left(R^{F, r}(t), t \geq 0\right)$ which are solution of :

$$
R_{t}=r+X_{t}+\int_{0}^{t} T F\left(R_{s}\right) d s, \quad t, r \geq 0,
$$

(recall that under $P_{0},\left(X_{t}\right)$ is a standard Brownian motion started at 0 ).

Proposition 5.1 Let $F \in \mathcal{H}, r \geq 0$. Then:

1. the process $\left(R^{F, r}(t), t \geq 0\right)$ takes its values in $\mathbb{R}_{+}, 0$ being a not-exit boundary and $R^{F, r}(t)$ goes to $+\infty$ as $t \rightarrow \infty$;

2. the r.v. $J_{0}^{R^{F, r}}=\inf _{t \geq 0} R^{F, r}(t)$ is finite.

(a) If $F \in \mathcal{H}^{\infty}$, then the density function of $J_{0}^{R^{F, r}}$ is

$$
\frac{1}{\int_{0}^{r} e^{2 F(y)} d y} e^{2 F(x)} 1_{[0, r]}(x) .
$$


(b) If $F$ does not belong to $\mathcal{H}^{\infty}$ then $P_{0}\left(J_{0}^{R^{F, r}}<a\right)=h(a) / h(r)$ for any a $\left.\in\right] 0, r[$ where :

$$
h(a):=\frac{\int_{0}^{a} e^{2 F(y)} d y}{\int_{a}^{\infty} e^{2 F(y)} d y}, \quad 0<a \leq r .
$$

Remark 5.2 1. Suppose that $F$ may be extended at 0 such that $F^{\prime}$ is right-continuous at 0 . Then $T F(x) \sim 1 / x$ as $x \rightarrow 0_{+}$. This means that, heuristically, $\left(R_{t}^{F, r}\right)$ and the three dimensional Bessel process have the same local behavior in the vicinity of 0 .

2. Let $F_{a}(x):=a \ln x, x>0$. Then $F_{a} \in \mathcal{H}$ iff $a>-1 / 2$. In this case $T F_{a}(x)=\frac{a+1}{x}, x>0$ and therefore $\left(R_{t}^{F_{a}, r}\right)$ is the Bessel process with dimension $2 a+3>2$.

Note that if $a=-1 / 2$, then $\left(R_{t}^{F_{-1 / 2}, r}\right)$ is the two dimensional Bessel process.

Proof of Proposition 5.1 1) The drift coefficient of $\left(R^{F, r}(t), t \geq 0\right)$ is singular at 0 . Following the classical boundary classification of one dimensional diffusions (see for instance [5], Chap II) we claim that 0 is a not-exit boundary.

Let $m(d x)=m(x) d x$ be the speed measure, and $s$ a scale function., which applying formulae given p. 17 in [5] we take as :

$$
\begin{aligned}
& s(x)=-\frac{1}{\int_{0}^{x} e^{2 F(y)} d y}, \quad x>0, \\
& m(x)=2 e^{-2 F(x)}\left(\int_{0}^{x} e^{2 F(y)} d y\right)^{2}, \quad x>0 .
\end{aligned}
$$

0 is a not-exit boundary, because :

$$
\int_{0}^{z}\left(\int_{a}^{z} m(y) d y\right) s^{\prime}(a) d a=\int_{0}^{z} m(y)\left(s(y)-s\left(0_{+}\right)\right) d y=\infty, \quad \forall z>0 .
$$

2) Since $s$ is a scale function of the process $\left(R^{F, r}(t), t \geq 0\right)$, then :

$$
P_{0}\left(T_{a}<T_{b}\right)=\frac{s(b)-s(r)}{s(b)-s(a)}, \quad 0<a<r<b,
$$

where $T_{c}$ is the first hitting time of level $\mathrm{c}$ for $\left(R^{F, r}(t), t \geq 0\right)$.

Since 0 is not-exit boundary, then $P_{0}\left(T_{0}<\infty\right)=0$.

Letting $a \rightarrow 0$ and $b \rightarrow \infty$, we get :

$$
P_{0}\left(T_{b}<\infty\right)=1, \quad P_{0}\left(T_{a}<\infty\right)=\frac{s(\infty)-s(r)}{s(\infty)-s(a)}<1, \quad 0<a<r<b .
$$

These relations imply that $R^{F, r}(t)$ drifts to $\infty$ as $t \rightarrow \infty$.

Note that the second identity may be interpreted as follows :

$$
P_{0}\left(T_{a}<\infty\right)=P_{0}\left(J_{\infty}^{R^{F, r}}<a\right)=\frac{s(\infty)-s(r)}{s(\infty)-s(a)}, \quad 0<a<r .
$$

If $F$ belongs to $\mathcal{H}^{\infty}$, then $s(\infty)=0$, and therefore the previous identity reduces to :

$$
P_{0}\left(J_{\infty}^{R^{F, r}}<a\right)=\frac{s(r)}{s(a)}=\frac{1}{\int_{0}^{r} e^{2 F(y)} d y} \int_{0}^{a} e^{2 F(y)} d y \quad 0<a<z,
$$

and then proves (5.5). 
Remark 5.3 Thanks to [5], Chap II), 0 is an entrance boundary iff $\int_{0}^{z}(s(z)-s(a)) m(a) d a<\infty$. Since $s\left(0_{+}\right)=-\infty$, the previous condition is equivalent to $\int_{0}^{z}(-s(a)) m(a) d a<\infty$. Relations (5.7) and (5.8) imply that 0 is an entrance boundary iff :

$$
\int_{0}^{1} e^{-2 F(x)}\left(\int_{0}^{x} e^{2 F(y)} d y\right) d x<\infty
$$

If the latter condition is satisfied, then 0 is an entrance and not-exit boundary. In particular:

- $\left(R^{F, 0}(t), t \geq 0\right)$ is well defined;

- if $r>0,\left(R^{F, r}(t), t \geq 0\right)$ never reaches level 0 .

Theorem 5.4 Let $F$ be in $\mathcal{H}$, satisfying (5.9) and $\left(Y_{t}\right)$ be the solution of :

$$
Y_{t}=X_{t}+\int_{0}^{t} F^{\prime}\left(2 S_{u}^{Y}-Y_{u}\right) d u, \quad t \geq 0 .
$$

Then

1. $\left(R_{t}^{Y}:=2 S_{t}^{Y}-Y_{t}\right)_{t \geq 0}$ is distributed as $\left(R^{F, 0}(t)\right)_{t \geq 0}$;

2. The processes $\left(S_{t}^{Y}\right)_{t \geq 0}$ and $\left(J_{t}^{R^{Y}}:=\inf _{u \geq t} R_{u}^{Y}\right)_{t \geq 0}$ are equal;

3. the following projection identity holds :

$$
E_{0}\left[f\left(S_{t}^{Y}\right) \mid \mathcal{R}_{t}^{Y}\right]=\Lambda^{F} f\left(R_{t}^{Y}\right), \quad t \geq 0,
$$

where

$$
\Lambda^{F} f(r)=\frac{\int_{0}^{r} f(y) e^{2 F(y)} d y}{\int_{0}^{r} e^{2 F(y)} d y}, \quad r>0 .
$$

Proof. 1) In a first step we compute expectations under $P_{a}$, where $a>0$ and we will take the limit $a \rightarrow 0$ later.

Let us introduce the Girsanov density $M_{t}^{F}$ of the law of $\left(Y_{u}, u \leq t\right)$ with respect to the Wiener measure $P_{a}$, restricted to $\mathcal{F}_{t}$ :

$$
M_{t}^{F}=\exp \left\{\int_{0}^{t} F^{\prime}\left(2 S_{u}^{X}-X_{u}\right) d X_{u}-\frac{1}{2} \int_{0}^{t} F^{\prime 2}\left(2 S_{u}^{X}-X_{u}\right) d u\right\}, \quad t \geq 0 .
$$

Consequently :

$$
E_{a}\left[G\left(R_{u}^{Y}, u \leq t\right)\right]=E_{a}\left[G\left(R_{u}^{X}, u \leq t\right) M_{t}^{F}\right],
$$

where $G$ is a non-negative functional.

Using Itô's formula (under $P_{a}$ ) we get :

$$
\begin{aligned}
F\left(2 S_{t}^{X}-X_{t}\right) & =F(a)-\int_{0}^{t} F^{\prime}\left(2 S_{u}^{X}-X_{u}\right) d X_{u}+2 \int_{0}^{t} F^{\prime}\left(2 S_{u}^{X}-X_{u}\right) d S_{u}^{X}+\frac{1}{2} \int_{0}^{t} F^{\prime \prime}\left(2 S_{u}^{X}-X_{u}\right) d u \\
& =-F(a)-\int_{0}^{t} F^{\prime}\left(R_{u}^{X}\right) d X_{u}+2 F\left(S_{t}^{X}\right)+\frac{1}{2} \int_{0}^{t} F^{\prime \prime}\left(R_{u}^{X}\right) d u .
\end{aligned}
$$

Hence

$$
M_{t}^{F}=e^{2 F\left(S_{t}^{X}\right)} \exp \left\{-F\left(R_{t}^{X}\right)-F(a)+\frac{1}{2} \int_{0}^{t}\left(F^{\prime \prime}-F^{2}\right)\left(R_{u}^{X}\right) d u\right\},
$$


and,

$$
E_{a}\left[G\left(R_{u}^{Y}, u \leq t\right)\right]=E_{a}\left[G\left(R_{u}^{X}, u \leq t\right) \exp \left\{-F\left(R_{t}^{X}\right)-F(a)+\frac{1}{2} \int_{0}^{t}\left(F^{\prime \prime}-F^{\prime 2}\right)\left(R_{u}^{X}\right) d u\right\} e^{2 F\left(S_{t}^{X}\right)}\right] .
$$

This implies that :

$$
\begin{aligned}
& E_{a}\left[G\left(R_{u}^{Y}, u \leq t\right)\right]=E_{0}\left[G\left(a+R_{u}^{X}, u \leq t\right)\right. \exp \left\{-F\left(a+R_{t}^{X}\right)-F(a)+\frac{1}{2} \int_{0}^{t}\left(F^{\prime \prime}-F^{\prime 2}\right)\left(a+R_{u}^{X}\right) d u\right\} \\
&\left.\times E_{0}\left[e^{2 F\left(a+S_{t}^{X}\right)} \mid \mathcal{R}_{t}^{X}\right]\right] .
\end{aligned}
$$

According to the projection formula (1.4), we have :

$$
E_{0}\left[e^{2 F\left(a+S_{t}^{X}\right)} \mid \mathcal{R}_{t}^{X}\right]=\frac{1}{R_{t}^{X}} \int_{0}^{R_{t}^{X}} e^{2 F(a+y)} d y .
$$

Let us introduce $F_{a}(y):=F(a+y), y \geq 0$ and :

$$
\tilde{F}_{a}(r):=e^{-F_{a}(r)} \int_{0}^{r} e^{2 F_{a}(y)} d y, r>0 .
$$

Taking the two first derivatives we get :

$$
\tilde{F}_{a}^{\prime}(r)=-F_{a}^{\prime}(r) \tilde{F}_{a}(r)+e^{F_{a}(r)}, \quad \tilde{F}_{a}^{\prime \prime}(r)=-\left(F_{a}^{\prime \prime}(r)-F_{a}^{\prime}(r)^{2}\right) \tilde{F}_{a}(r), \quad r>0 .
$$

Finally we have :

$$
E_{a}\left[G\left(R_{u}^{Y}, u \leq t\right)\right]=E_{0}\left[G\left(a+R_{u}^{X}, u \leq t\right) N_{t}^{F}\right],
$$

where $\left(N_{t}^{F}\right)$ is the non-negative $\left(\left(\mathcal{R}_{t}^{X}\right), P_{0}\right)$ martingale :

$$
N_{t}^{F}=e^{-F_{a}(0)} \frac{\tilde{F}_{a}\left(R_{t}^{X}\right)}{R_{t}^{X}} \exp \left\{-\frac{1}{2} \int_{0}^{t} \frac{\tilde{F}_{a}^{\prime \prime}}{\tilde{F}_{a}}\left(R_{u}^{X}\right) d u\right\} .
$$

The process $\left(R_{t}^{X}\right)$ being a three dimensional Bessel process, there exists a $P_{0}$-Brownian motion $\left(\beta_{t}\right)$ such that:

$$
R_{t}^{X}=\beta_{t}+\int_{0}^{t} \frac{1}{R_{u}^{X}} d u .
$$

Let $H_{a}$ be the function : $H_{a}(x)=\tilde{F}_{a}(x) / x, x>0$. Since $\left(N_{t}^{F}\right)$ is a $P_{0}$ martingale, Itô's formula implies that :

$$
N_{t}^{F}=1+e^{-F_{a}(0)} \int_{0}^{t} H_{a}^{\prime}\left(R_{s}^{X}\right) \exp \left\{-\frac{1}{2} \int_{0}^{s} \frac{\tilde{F}_{a}^{\prime \prime}}{\tilde{F}_{a}}\left(R_{u}^{X}\right) d u\right\} d \beta_{s} .
$$

It turns out that $\left(N_{t}^{F}\right)$ may be written as an exponential martingale with respect to the Brownian motion $\left(\beta_{t}\right)$ :

$$
N_{t}^{F}=\exp \left\{\int_{0}^{t} \xi_{s} d \beta_{s}-\frac{1}{2} \int_{0}^{t} \xi_{s}^{2} d s\right\}
$$

with :

$$
\xi_{t}=\frac{H_{a}^{\prime}\left(R_{t}^{X}\right) R_{t}^{X}}{\tilde{F}_{a}\left(R_{t}^{X}\right)}=\frac{H_{a}^{\prime}\left(R_{t}^{X}\right)}{H_{a}\left(R_{t}^{X}\right)} .
$$

Using (5.14), and after some easy calculations, we obtain :

$$
\begin{aligned}
\frac{H_{a}^{\prime}(r)}{H_{a}(r)}=\frac{H_{a}^{\prime}(r) r}{\tilde{F}_{a}(r)} & =-F_{a}^{\prime}(r)+\frac{e^{F_{a}(r)}}{\tilde{F}_{a}(r)}-\frac{1}{r} \\
& =T F_{a}(r)-\frac{1}{r}, \quad r>0 .
\end{aligned}
$$


Let us introduce the following p.m. $\mathbb{Q}^{F}$ on $\left(\Omega, \mathcal{F}_{\infty}\right)$ :

$$
\mathbb{Q}^{F}\left(\Gamma_{t}\right)=E_{0}\left[1_{\Gamma_{t}} N_{t}^{F}\right], \quad \forall t \geq 0, \Gamma_{t} \in \mathcal{F}_{t} .
$$

This allows us to write (5.15) in the following form :

$$
E_{a}\left[G\left(R_{u}^{Y}, u \leq t\right)\right]=\mathbb{Q}^{F}\left[G\left(a+R_{u}^{X}, u \leq t\right)\right] .
$$

From Girsanov 's theorem, there exists a $\mathbb{Q}^{F}$ Brownian motion $\left(\bar{\beta}_{t}\right)$ such that :

$$
\beta_{t}=\bar{\beta}_{t}+\int_{0}^{t} \xi_{s} d s
$$

Consequently :

$$
R_{t}^{X}=\beta_{t}+\int_{0}^{t} T F_{a}\left(R_{u}^{X}\right) d u .
$$

Taking the limit $a \rightarrow 0$, we obtain 1. of Theorem 5.1.

2) Point 2. follows from the fact that $R^{F, 0}(t)$ drifts to $\infty$ as $t \rightarrow \infty$.

3) Mimicking the approach developed in point 1) above we obtain :

$$
\begin{aligned}
E_{a}\left[G\left(R_{u}^{Y}, u \leq t\right) g\left(S_{t}^{Y}\right)\right]=E_{0}\left[G\left(a+R_{u}^{X}, u \leq t\right) \exp \left\{-F\left(a+R_{t}^{X}\right)-F(a)+\frac{1}{2} \int_{0}^{t}\left(F^{\prime \prime}-F^{\prime 2}\right)\left(a+R_{u}^{X}\right) d u\right\}\right. \\
\left.\times E_{0}\left[g\left(S_{t}^{X}\right) e^{2 F\left(a+S_{t}^{X}\right)} \mid \mathcal{R}_{t}^{X}\right]\right]
\end{aligned}
$$

where $g: \mathbb{R}_{+} \rightarrow \mathbb{R}_{+}$is a Borel function.

The projection property (1.4) implies (5.11).

Remark 5.5 Similar computations relating Ray-Knight theorems and the Ricatti equations are developed in [7].

\subsection{About the non-injectivity property of the operator $T$}

Now, we point out that the operator $T$ is not one-to-one. This lack of injectivity has an important consequence : it is possible to construct a family of processes $\left(Y_{t}\right)$ such that the associated processes $\left(R_{t}^{Y}\right)$ are Markovian, with the same infinitesimal generator (see Theorem 5.8).

Let $U_{\alpha}(F)$ be the function :

$$
U_{\alpha}(F)(x):=F(x)-\ln \left(1+\alpha \int_{0}^{x} e^{2 F(y)} d y\right), \quad x>0,
$$

where $F \in \mathcal{H}$ and $\alpha \in \mathbb{R}$ satisfy :

$$
\alpha \geq-\frac{1}{\int_{0}^{\infty} e^{2 F(y)} d y},
$$

with the convention : $1 / \infty=0$.

Note that $U_{0}$ is the identity operator.

The following lemma shows that the operator $T$ is not one-to-one.

Lemma 5.6 Assume $F \in \mathcal{H}$.

1. Let $\alpha$ satisfy (5.19), then $U_{\alpha}(F) \in \mathcal{H}$. 
2. Let $G \in \mathcal{H}$. Then $T G=T F$ iff $G=c+U_{\alpha}(F)$, for some $c \in \mathbb{R}$ and $\alpha$ satisfying (5.19).

Proof. 1) Let $A F$ be the function :

$$
A F(x)=\int_{0}^{x} e^{2 F(y)} d y, x \geq 0 .
$$

It is clear that (5.19) implies :

$$
1+\alpha A F(x)=1+\alpha \int_{0}^{x} e^{2 F(y)} d y>0, \quad x \geq 0 .
$$

From the definition of $U_{\alpha}(F)$, we have :

$$
\int_{0}^{x} e^{2 U_{\alpha}(F)(y)} d y=\int_{0}^{x} \frac{A F^{\prime}(y)}{(1+\alpha A F(y))^{2}} d y=\frac{A F(x)}{1+\alpha A F(x)}<\infty, \quad x \geq 0 .
$$

This implies that $U_{\alpha}(F) \in \mathcal{H}$.

2) a) First we observe that :

$$
T F(x)=\left(-F(x)+\ln \left(\int_{0}^{x} e^{2 F(y)} d y\right)\right)^{\prime}, \quad x \geq 0 .
$$

Let $G \in \mathcal{H}$. Then $T G=T F$ iff

$$
G(x)-\ln \left(\int_{0}^{x} e^{2 G(y)} d y\right)=F(x)-\ln \left(\int_{0}^{x} e^{2 F(y)} d y\right)+c_{1}, \quad x \geq 0 .
$$

Multiplying both sides by 2 , and exponentiating, we get

$$
\frac{e^{2 G(x)}}{\left(\int_{0}^{x} e^{2 G(y)} d y\right)^{2}}=c_{2} \frac{e^{2 F(x)}}{\left(\int_{0}^{x} e^{2 F(y)} d y\right)^{2}}, \quad x \geq 0
$$

with $c_{2}=e^{2 c_{1}}>0$.

b) Integrating (5.23) over $[x, \infty[$ we obtain :

$$
\begin{aligned}
\frac{1}{\int_{0}^{x} e^{2 G(y)} d y} & =\frac{c_{2}}{\int_{0}^{x} e^{2 F(y)} d y}-\frac{c_{2}}{\int_{0}^{\infty} e^{2 F(y)} d y}+\frac{1}{\int_{0}^{\infty} e^{2 G(y)} d y} \\
& =\frac{c_{2}}{\int_{0}^{x} e^{2 F(y)} d y}+c_{3} .
\end{aligned}
$$

Hence,

$$
\int_{0}^{x} e^{2 G(y)} d y=\frac{\int_{0}^{x} e^{2 F(y)} d y}{c_{2}+c_{3} \int_{0}^{x} e^{2 F(y)} d y} .
$$

Taking derivatives of both sides, we obtain :

$$
\begin{gathered}
e^{2 G(x)}=\frac{c_{2} e^{2 F(x)}}{\left(c_{2}+c_{3} \int_{0}^{x} e^{2 F(y)} d y\right)^{2}}, \\
G(x)=F(x)-\ln \left(1+\alpha \int_{0}^{x} e^{2 F(y)} d y\right)-\frac{1}{2} \ln c_{2}=U_{\alpha}(F)(x)+c,
\end{gathered}
$$

with $\alpha=c_{3} / c_{2}$.

The identity (5.24) implies that the real number $\alpha$ satisfies (5.21)(this relation being equivalent to $(5.19))$ 
c) Conversely let $G=c+U_{\alpha}(F)$, with $F \in \mathcal{H}$. According to part 1. of Lemma 5.6, $G$ belongs to $\mathcal{H}$, and we have :

$$
\int_{0}^{x} e^{2 G(y)} d y=e^{2 c} \int_{0}^{x} \frac{e^{2 F(y)}}{\left(1+\alpha \int_{0}^{y} e^{2 F(z)} d z\right)^{2}} d y=e^{2 c} \frac{\int_{0}^{x} e^{2 F(y)} d y}{1+\alpha \int_{0}^{x} e^{2 F(y)} d y}
$$

Therefore :

$$
\frac{1}{\int_{0}^{x} e^{2 G(y)} d y}=\frac{e^{-2 c}}{\int_{0}^{x} e^{2 F(y)} d y}+\alpha e^{-2 c} .
$$

Taking the derivative, we obtain a relation of the type (5.23). In step a) above, we have proved that this property is equivalent to $T G=T F$.

Remark 5.7 In fact, these exponential transforms have already been introduced in [14], [15], in the discussion of these authors' extension of Pitman's theorem which we have recalled at the end of the above Section 1.

Our present discussion, in subsection 5.1, is the deterministic counterpart of that in [14], [15], where $F$ in (5.18) was replaced by Brownian motion with drift.

Lemma 5.6 and Theorem 5.4 taken together imply the following consequence.

Theorem 5.8 Let $F$ be in $\mathcal{H}$ satisfying (5.9), $\alpha$ such that (5.19) holds, and $\left(Y_{t}^{\alpha}\right)$ be the solution of:

$$
Y_{t}=X_{t}+\int_{0}^{t} U_{\alpha}(F)^{\prime}\left(2 S_{u}^{Y}-Y_{u}\right) d u, \quad t \geq 0
$$

Then the law of $\left(R_{t}^{Y^{\alpha}}\right)_{t \geq 0}$ does not depend on $\alpha$, and is equal to the distribution of $\left(R^{F, 0}(t)\right)_{t \geq 0}$.

Example 5.9 Let $F(x)=\lambda, x>0$. Then

$$
U_{\alpha}(F)(x)=\lambda-\ln \left(1+\alpha e^{2 \lambda} x\right), \quad x \geq 0,
$$

and $T F(x)=1 / x, x>0$.

Therefore let $Y^{\alpha}$ be the solution of

$$
Y_{t}=X_{t}-\int_{0}^{t} \frac{\alpha e^{2 \lambda}}{1+\alpha e^{2 \lambda}\left(2 S_{u}^{Y}-Y_{u}\right)} d u
$$

then $\left(R_{t}^{Y^{\alpha}}\right)_{t \geq 0}$ is distributed as a three dimensional Bessel process started at 0 .

Some elementary computation shows that $Y^{\alpha}$ is distributed as the max-diffusion solution of (2.11), with

$$
g(x)=\frac{\alpha}{\alpha x+e^{-2 \lambda}}, \quad x \geq 0 .
$$

It is possible to choose $\alpha=\infty$ in (5.27). In this case $Y^{\infty}$ solves :

$$
Y_{t}=X_{t}-\int_{0}^{t} \frac{1}{2 S_{u}^{Y}-Y_{u}} d u
$$

and is associated with $g(x)=1 / x$. 
Example 5.10 Let $F(x)=\lambda x, x>0$. Then :

$$
U_{\alpha}(F)(x)=\lambda x-\ln \left(1+\alpha \frac{e^{2 \lambda x}-1}{2 \lambda}\right), \quad T F(x)=\lambda \operatorname{coth}(\lambda x) \quad x>0 ;
$$

$Y^{\alpha}$ is the solution of

$$
Y_{t}=X_{t}+\int_{0}^{t}\left(\lambda-\frac{2 \lambda \alpha e^{2 \lambda\left(2 S_{u}^{Y}-Y_{u}\right)}}{2 \lambda+\alpha\left(e^{2 \lambda\left(2 S_{u}^{Y}-Y_{u}\right)}-1\right)}\right) d u
$$

and $\left(R_{t}^{Y^{\alpha}}\right)_{t \geq 0}$ is distributed as the solution of

$$
R_{t}=X_{t}+\lambda \int_{0}^{t} \operatorname{coth}\left(\lambda R_{u}\right) d u
$$

\subsection{A converse to Theorem 5.4}

Let us introduce some new notation :

$$
\widehat{\mathcal{H}}=\{G:] 0, \infty\left[\mapsto \mathbb{R}, G \text { of class } C^{1}, \int_{x}^{\infty} e^{-2 G(y)} d y<\infty, \forall x>0 \text { and } \int_{0}^{\infty} e^{-2 G(y)} d y=\infty\right\},
$$

and

$$
C(G)(x):=-G(x)+\ln \left(\int_{0}^{x} e^{2 G(y)} d y\right), \quad x>0, G \in \mathcal{H} .
$$

To present a converse to Theorem 5.4 we establish the following preliminary lemma.

Lemma 5.11 1. Let $F \in \mathcal{H}$. Then $C(F) \in \widehat{\mathcal{H}}$ and $T(F)=C(F)^{\prime}$.

2. Let $G \in \widehat{\mathcal{H}}$ and $F \in \mathcal{H}$. Then the following are equivalent :

(a) $T(F)=G^{\prime}$;

(b) there exist $c \in \mathbb{R}$ and $\alpha \geq 0$ such that :

$$
F(x)=c-G(x)-\ln \left(\alpha+\int_{x}^{\infty} e^{-2 G(y)} d y\right), \quad x>0,
$$

(c) there exist $c, \rho \in \mathbb{R}$ such that $F=c+U_{\rho}\left(F_{0}\right)$ where:

$$
F_{0}(x)=-G(x)-\ln \left(\int_{x}^{\infty} e^{-2 G(y)} d y\right), \quad x>0 .
$$

Proof. 1) We have :

$$
\int_{x}^{\infty} e^{-2 C(F)(y)} d y=\int_{x}^{\infty} \frac{e^{2 F(y)}}{\left(\int_{0}^{y} e^{2 F(z)} d z\right)^{2}} d y=\frac{1}{\int_{0}^{x} e^{2 F(y)} d y}-\frac{1}{\int_{0}^{\infty} e^{2 F(y)} d y}<\infty .
$$

Consequently $C(F) \in \widehat{\mathcal{H}}$.

It is clear that $T(F)=C(F)^{\prime}$.

2) Let $G \in \widehat{\mathcal{H}}$ and $F \in \mathcal{H}$.

Using relation (5.22) and proceeding analogously to the proof of Lemma 5.6, shows that $T(F)=G^{\prime}$ iff :

$$
\frac{e^{2 F(x)}}{\left(\int_{0}^{x} e^{2 F(y)} d y\right)^{2}}=c_{2} e^{-2 G(x)}, \quad x \geq 0,
$$


Mimicking the approach developed in step 2) a) of the proof of Lemma 5.6, we obtain successively :

$$
\begin{gathered}
\frac{1}{\int_{0}^{x} e^{2 F(y)} d y}=c_{3}+c_{2} \int_{x}^{\infty} e^{-2 G(y)} d y, \quad\left(c_{2}>0, c_{3} \geq 0\right), \\
\int_{0}^{x} e^{2 F(y)} d y=\frac{1}{c_{3}+c_{2} \int_{x}^{\infty} e^{-2 G(y)} d y}, \\
e^{2 F(x)}=\frac{e^{-2 G(x)}}{\left(c_{3}+c_{2} \int_{x}^{\infty} e^{-2 G(y)} d y\right)^{2}}, \\
F(x)=c-G(x)-\ln \left(\alpha+\int_{x}^{\infty} e^{-2 G(y)} d y\right) .
\end{gathered}
$$

This proves $(a) \Rightarrow(b)$.

The proof of $(b) \Rightarrow(a)$ is a direct consequence of previous calculations and $\int_{0}^{\infty} e^{-2 G(y)} d y=\infty$.

Lemma 5.6 implies that $(a) \Leftrightarrow(c)$.

Theorem 5.12 Let $G$ be a function in $\widehat{\mathcal{H}}$ and $\left(R_{t}\right)$ the diffusion process taking its values in $\mathbb{R}_{+}$ solution of :

$$
R_{t}=X_{t}+\int_{0}^{t} G^{\prime}\left(R_{s}\right) d s, \quad t \geq 0 .
$$

Then :

1. $R_{t}$ goes to $\infty$ as $t \rightarrow \infty$.

2. Let $J_{t}^{R}=\inf _{s \geq t} R_{s}$ and $Y_{t}:=2 J_{t}^{R}-R_{t}, t \geq 0$.

(a) $S_{t}^{Y}=\sup _{s \leq t} Y_{s}=J_{t}^{R}$ and $R_{t}=2 S_{t}^{Y}-Y_{t}, t \geq 0$;

(b) there exists a Brownian motion $\left(B_{t}\right)$ starting at 0 such that :

$$
Y_{t}=B_{t}+\int_{0}^{t} F_{0}^{\prime}\left(2 S_{u}^{Y}-Y_{u}\right) d u
$$

where $F_{0}$ is the function defined by (5.36).

Proof. 1) From Lemma 5.11, there exist functions $F \in \mathcal{H}$ such that $T(F)=G^{\prime}$. Consequently part 1. of Theorem 5.12 is a direct consequence of Proposition 5.1, 1.

It is clear that 1. implies 2. (a).

2) Let us define $: s(x)=-\int_{x}^{\infty} e^{-2 G(y)} d y, x>0$. We have :

$$
s^{\prime}(x)=e^{-2 G(x)}, \quad s^{\prime \prime}(x)=-2 G^{\prime}(x) e^{-2 G(x)}=-2 G^{\prime}(x) s^{\prime}(x), x>0 .
$$

Consequently $\frac{1}{2} s^{\prime \prime}(x)+G^{\prime}(x) s^{\prime}(x)=0$; hence $s$ is a scale function for $\left(R_{t}\right)$.

It is obvious from the definition of $s$ that $s\left(0_{+}\right)=-\infty$ and $s(\infty)=0$. Therefore, applying the result of Saisho and Tanemura ([31] or [35] theorem 12.7) : there exists a $\left(\sigma\left(R_{s}, s \leq t\right)\right)_{t>0}$ Brownian motion $\left(B_{t}\right)$ started at 0 such that

$$
R_{t}=-B_{t}+\int_{0}^{t}\left(\frac{s^{\prime}}{s}-\frac{s^{\prime \prime}}{2 s^{\prime}}\right)\left(R_{u}\right) d u+2 J_{t}^{R} .
$$


Using (5.40) and (5.36) give :

$$
\frac{s^{\prime}(x)}{s(x)}-\frac{s^{\prime \prime}(x)}{2 s^{\prime}(x)}=-\frac{e^{-2 G(x)}}{\int_{x}^{\infty} e^{-2 G(y)} d y}+G^{\prime}(x)=-F_{0}^{\prime}(x) .
$$

As a result :

$$
Y_{t}=2 J_{t}^{R}-R_{t}=B_{t}+\int_{0}^{t} F_{0}^{\prime}\left(2 S_{u}^{Y}-Y_{u}\right) d u
$$

\section{Conclusion}

In this paper, we have shown that the classical theorem of Pitman concerning his representation of $B E S(3)$ as $2 S_{t}-B_{t}, t \geq 0$, where $S_{t}=\sup _{s \leq t} B_{s}$, extends when the Brownian motion $B$ is replaced by a process distributed as a limiting law of certain penalization procedures of Brownian motion.

Similar extensions are also obtained, starting with the Ray-Knight theorems for Brownian local times. On the other hand, one will not find in this paper any new non-invertible, strictly adapted, transform of Brownian motion, or of related processes, with a remarkable distribution, such as in MatsumotoYor $([14],[15])$, who deal with exponential functionals, or Bertoin [2] who discusses some variants of Pitman's theorem for a class of Lévy processes, or again Biane, Bougerol and O'Connell [3] who obtain multidimensional extensions of Pitman's theorem.

\section{References}

[1] J. Azéma and M. Yor. Une solution simple au problème de Skorokhod. In Séminaire de Probabilités, XIII (Univ. Strasbourg, Strasbourg, 1977/78), volume 721 of Lecture Notes in Math., pages 90-115. Springer, Berlin, 1979.

[2] J. Bertoin. An extension of Pitman's theorem for spectrally positive Lévy processes. Ann. Probab., 20(3):1464-1483, 1992.

[3] P. Biane, P. Bougerol, and N. O'Connell. Brownian paths and Littlemann paths. To appear in Duke Math. Journal, 2005.

[4] Ph. Biane. Intertwining of Markov semi-groups, some examples. In Séminaire de Probabilités, XXIX, volume 1613 of Lecture Notes in Math., pages 30-36. Springer, Berlin, 1995.

[5] A. N. Borodin and P. Salminen. Handbook of Brownian motion-facts and formulae. Probability and its Applications. Birkhäuser Verlag, Basel, second edition, 2002.

[6] Ph. Carmona, F. Petit, and M. Yor. Beta-gamma random variables and intertwining relations between certain Markov processes. Rev. Mat. Iberoamericana, 14(2):311-367, 1998.

[7] C. Donati-Martin and M. Yor. Some Brownian functionals and their laws. Ann. Probab., 25(3):1011-1058, 1997.

[8] T. Jeulin. Un théorème de J. W. Pitman. In Séminaire de Probabilités, XIII (Univ. Strasbourg, Strasbourg, 1977/78), volume 721 of Lecture Notes in Math., pages 521-532. Springer, Berlin, 1979. With an appendix by M. Yor.

[9] T. Jeulin and M. Yor. Autour d'un Théorème de Ray. In Temps locaux, volume 52-53 of Astérisque, pages 145-158. Société Mathématique de France, 1978.

[10] T. Jeulin and M. Yor. Sur les distributions de certaines fonctionnelles du mouvement brownien. In Seminar on Probability, XV (Univ. Strasbourg, Strasbourg, 1979/1980) (French), volume 850 of Lecture Notes in Math., pages 210-226. Springer, Berlin, 1981. 
[11] D. P. Kennedy. Some martingales related to cumulative sum tests and single-server queues. Stochastic Processes Appl., 4(3):261-269, 1976.

[12] F. B. Knight. On the sojourn times of killed Brownian motion. In Séminaire de Probabilités, XII (Univ. Strasbourg, Strasbourg, 1976/1977), volume 649 of Lecture Notes in Math., pages 428-445. Springer, Berlin, 1978.

[13] H. Matsumoto and M. Yor. A version of Pitman's $2 M-X$ theorem for geometric Brownian motions. C. R. Acad. Sci. Paris Sér. I Math., 328(11):1067-1074, 1999.

[14] H. Matsumoto and M. Yor. An analogue of Pitman's $2 M-X$ theorem for exponential Wiener functionals. I. A time-inversion approach. Nagoya Math. J., 159:125-166, 2000.

[15] H. Matsumoto and M. Yor. An analogue of Pitman's $2 M-X$ theorem for exponential Wiener functionals. II. The role of the generalized inverse Gaussian laws. Nagoya Math. J., 162:65-86, 2001.

[16] H. Matsumoto and M. Yor. Exponential Functionals of Brownian motion. I : Probability laws at fixed time. Probability Surveys, 2005/2006.

[17] H. Matsumoto and M. Yor. Exponential Functionals of Brownian motion. II : Some related diffusion processes. Probability Surveys, 2005/2006.

[18] H. Miyazaki and H. Tanaka. A theorem of Pitman type for simple random walks on $\mathbf{Z}^{d}$. Tokyo J. Math., 12(1):235-240, 1989.

[19] J. Obłój. The Skorokhod embedding problem and its offspring. Probab. Surv., 1:321-390 (electronic), 2004.

[20] J. Obłój. The Skorokhod embedding problem and some families of Brownian martingales. Thèse Paris VI-Varsovie, 2005.

[21] J. Obłój. A complete characterization of local martingales which are function of Brownian motion and its maximum. To appear in Séminaire de Probabilités, XL, 2006.

[22] J. Pitman and M. Yor. A decomposition of Bessel bridges. Z. Wahrsch. Verw. Gebiete, 59(4):425$457,1982$.

[23] J. W. Pitman. One-dimensional Brownian motion and the three-dimensional Bessel process. Advances in Appl. Probability, 7(3):511-526, 1975.

[24] B. Rauscher. Some remarks on Pitman's theorem. In Séminaire de Probabilités, XXXI, volume 1655 of Lecture Notes in Math., pages 266-271. Springer, Berlin, 1997.

[25] D. Revuz and M. Yor. Continuous martingales and Brownian motion, volume 293 of Grundlehren der Mathematischen Wissenschaften [Fundamental Principles of Mathematical Sciences]. Springer-Verlag, Berlin, third edition, 1999.

[26] L. C. G. Rogers. Characterizing all diffusions with the $2 M-X$ property. Ann. Probab., 9(4):561$572,1981$.

[27] L. C. G. Rogers and J. W. Pitman. Markov functions. Ann. Probab., 9(4):573-582, 1981.

[28] B. Roynette, P. Vallois, and M. Yor. Limiting laws associated with Brownian motion perturbed by its maximum, minimum and local time, II. To appear in Studia Sci. Math. Hungar., 2005.

[29] B. Roynette, P. Vallois, and M. Yor. Limiting laws for long Brownian bridges perturbed by their one-sided maximum, III. Periodica Mathematica Hungarica, 50(1-2):247-280, 2005.

[30] B. Roynette, P. Vallois, and M. Yor. Pénalisations et extensions du théorème de Pitman, relatives au mouvement brownien et à son maximum unilatère. In Seminar on Probability, XXXIX (P.A. Meyer, in memoriam), Lecture Notes in Math. Springer, Berlin, 2006. 
[31] Y. Saisho and H. Tanemura. Pitman type theorem for one-dimensional diffusion processes. Tokyo J. Math., 13(2):429-440, 1990.

[32] K. Takaoka. On the martingales obtained by an extension due to Saisho, Tanemura and Yor of Pitman's theorem. In Séminaire de Probabilités, XXXI, volume 1655 of Lecture Notes in Math., pages 256-265. Springer, Berlin, 1997.

[33] H. Tanaka. Time reversal of random walks in one-dimension. Tokyo J. Math., 12(1):159-174, 1989.

[34] H. Tanaka. Time reversal of random walks in $\mathbf{R}^{d}$. Tokyo J. Math., 13(2):375-389, 1990.

[35] M. Yor. Some aspects of Brownian motion. Lectures in Mathematics ETH Zürich. Birkhäuser Verlag, Basel, 1997. Part II : Some recent martingale problems. 